\title{
Fault Diagnosis of Rolling Bearing based on an Improved Denoising Technique using Complete Ensemble Empirical Mode Decomposition and Adaptive Thresholding Method
}

\section{Prashant Kumar Sahu ( $\sim$ prashantphd1@iitkgp.ac.in )}

Indian Institute of Technology Kharagpur

\section{Rajiv Nandan Rai}

Indian Institute of Technology Kharagpur

\section{Research Article}

Keywords: CEEMD, ATD, Kurtosis, Envelope Spectrum, IMFs

Posted Date: February 9th, 2021

DOl: https://doi.org/10.21203/rs.3.rs-159429/v1

License: (c) (i) This work is licensed under a Creative Commons Attribution 4.0 International License. Read Full License

Version of Record: A version of this preprint was published at Journal of Vibration Engineering \& Technologies on June 24th, 2022. See the published version at https://doi.org/10.1007/s42417-02200591-z. 


\title{
Fault Diagnosis of Rolling Bearing based on an Improved Denoising Technique using Complete Ensemble Empirical Mode Decomposition and Adaptive Thresholding Method Prashant Kumar Sahu $^{1 *}$, Rajiv Nandan Rai ${ }^{1}$ \\ ${ }^{1}$ Subir Chowdhury School of Quality and Reliability, IIT Kharagpur, India *Corresponding author (Email: prashantphd1 @ iitkgp.ac.in, Tele: 03222283992 Mobile: +91-9110167703)
}

\begin{abstract}
The vibration signals for rotating machines are generally polluted by excessive noise and can lose the fault information at the early development phase. In this paper, an improved denoising technique is proposed for early faults diagnosis of rolling bearing based on the complete ensemble empirical mode decomposition (CEEMD) and adaptive thresholding (ATD) method. Firstly, the bearing vibration signals are decomposed into a set of various intrinsic mode functions (IMFs) using CEEMD algorithm. The IMFs grouping and selection are formed based upon the correlation coefficient value. The noise-predominant IMFs are subjected to adaptive thresholding for denoising and then added to the low-frequency IMFs for signal reconstruction. The effectiveness of the proposed method denoised signals are measured based on kurtosis value and the envelope spectrum analysis. The presented method results on experimental datasets illustrate that the

proposed approach is an effective denoising technique for early fault detection in the rolling bearing.
\end{abstract}

Keywords: CEEMD; ATD; Kurtosis; Envelope Spectrum; IMFs

\section{Introduction}

Rotating machinery performs a crucial part in various industrial and automobile applications. Rotating machines such as rolling bearings are vital components, which determine the operating states of the system. A defect in bearing, if remain undetected can lead to catastrophic failure of the machinery [1]. The monitoring of such components is necessary, as they directly affect the 
production and maintenance cost. Therefore, effective fault diagnosis of rotating machinery has been provided adequate attention by the research fraternity.

The analysis of vibration signals is a prominent technique which is extensively used in fault detection by monitoring the changes in the characteristic defect frequency of rotating machinery [2]. This measurement technique can be categorized as time-domain, frequency-domain, and timefrequency-domain. The simplest approach for fault diagnosis of rotating machine is by extracting the time-domain features such as mean, root mean square, kurtosis, crest factor etc. [3]. The kurtosis parameter is widely used in the bearing fault diagnosis. The kurtosis value for the healthy bearing is equal to or less than three and higher than three for the defective bearing, which produces the cycle of impact repeated periodically [4]. However, it does not define the nature and the location of the fault type. Therefore, another advanced technique is required, such as envelope spectrum analysis. Envelope analysis is an effective technique to obtain a characteristic defect frequency of the bearing. However, the vibration signals are generally polluted by excessive noise and can lose the bearing fault information in the early development phase. The early fault detection by conventional techniques such as kurtosis or envelope spectrum with noisy vibration signal becomes very difficult [5], [6]. Therefore, the denoising is required to enhance the signal quality and kurtosis parameter sensitivity, and the envelope spectrum performance.

The vibration signal excited by local defects of bearings shows non-stationary behavior. Empirical mode decomposition (EMD) proposed by Huang et al. [7] is an effective method for analyzing the non-stationary and nonlinear signals. It is widely used in the field of bearing fault diagnosis. Rai \& Mohanty utilize the Hilbert-Huang transform (HHT) or EMD for bearing fault diagnosis, which gives multi-resolution of signal in various frequency scales. They consider FFT analysis for each IMFs from the EMD process to enhance the Hilbert transform (HT) efficiency in the frequency domain [8]. Zhao et al. proposed an algorithm based on EMD and wavelet shrinkage, which accurately estimates the rolling bearing instantaneous rotational frequency. They decomposed the signals by EMD to produce a series of IMFs. The noise-dominant IMFs are then selected for denoising by soft thresholding and then added to the remaining IMFs for signal reconstruction. Their model shows better integrity of the signal, high signal to noise ratio, and highlights the fault features [9]. Effective IMFs are chosen according to the correlation coefficients values[10] and then are subjected to envelope analysis for signal demodulation for the early fault 
diagnosis of rolling bearings [11]. However, this technique is unstable because of the selection of too strong and weak IMFs. Abdelkader et al. proposed an effective method for bearing fault diagnosis. They utilize the EMD approach to decompose the signal into IMFs. The effective modes are selected based on the trip point, which is defined by analyzing each mode's energy content with respect to the white noise characteristics. The singular IMF is selected by comparing the average energy of selected IMFs with the unselected IMFs. Then, the singular selected IMF is subjected to optimized thresholding to obtain a denoised signal. Their method effectiveness is measured by the kurtosis and the envelope analysis [12][13].

However, the major drawbacks of the EMD process are mode mixing and aliasing [14][15]. To address the problem that arises in the EMD process, Wu and Huang proposed a new noiseassisted data analysis technique, commonly known as Ensemble empirical mode decomposition (EEMD) [15]. Yaguo Lei et al. [16] present the EEMD method application to rotor fault diagnosis in the rotating machinery. The EEMD methods provide better results as compared to EMD for fault diagnosis. To further improve the mode mixing, over decomposition, and residual noise in IMFs, an adaptive technique was proposed known as complete ensemble empirical mode decomposition (CEEMD). Meijiao Li et al. utilizes the CEEMD approach for fault diagnosis of rolling bearing [17]. Recently various work has been carried out using CEEMD and machine learning techniques for fault diagnosis and prognosis as well. Lu et al. analyze the defect severity of bearing using CEEMD and Kernal SVM [18]. Liu et al. propose a new method for fault diagnosis and classification based on energy moment feature using CEEMD and probabilistic neural network and particle swarm optimization, optimized by linear decreasing weight [19]. Recent work shows that the CEEMD algorithm is an effective signal decomposition method for fault information and feature extraction. Therefore, CEEMD is used in the presented work for the signal decomposition of bearing signal under the influence of white Gaussian noise.

In this work, to eliminate the selection of too strong and weak modes, IMFs grouping is formed based on the correlation coefficient value. The noise predominant and low-frequency informative IMFs are selected for further processing. The noise-predominant IMFs are then subjected to thresholding operation. In hard and soft thresholding, any values in the signal smaller than the threshold are considered noise and are further set to zero. However, some of the values are smaller than the threshold belongs to a useful part of the signals, and it should be retained to 
prevent the loss of local information about the bearing fault. The denoising of non-stationary vibration signals is commonly carried out by using the wavelet transform (WT) and the EMD approach [20]. In the wavelet transform, the appropriate selection of the mother wavelet and the decomposition level are two essential parameters for signal denoising. However, selecting these parameters are difficult task, and its results may have certain randomness [21][22]. In this work, Wavelet soft thresholding with symlet-2 and four levels of decomposition is used to denoise the selected IMFs and finally compared with ATD. The WTD thresholding shows better continuity of signal, but few periodic impulses are observed, missing which can lead to loss of information. So, To ease the thresholding operation, an adaptive thresholding method based on the $3 \sigma$ criterion for threshold value determination and peak filtering technique [23] is used in this work for retaining maximum fault information of the bearing.

In this paper, an improved denoising method is proposed for early fault detection of rolling bearing. The novelty of this work is the combination of the existing CEEMD decomposition method with adaptive thresholding and peaking filtering method for bearing fault diagnosis. This paper also defines the correlation coefficient-based threshold for the effective selection of bearing IMFs. The summary of work are as follows: Firstly, bearing signals are decomposed into various intrinsic modes of functions (IMFs) by complete ensemble empirical mode decomposition (CEEMD). IMFs grouping and selection are made based upon the correlation coefficient. Then, the selected noise predominant IMFs are de-noised by adaptive thresholding based on the $3 \sigma$ criterion for threshold value determination and peak filtering technique. Finally, the denoised signal is obtained by adding denoised IMFs and low-frequency IMFs and then subjected to envelope spectrum analysis to extract the rolling bearing fault frequencies.

The organization of this paper is as follows: Section 2 briefly introduces the signal decomposition method. In Section 3, the proposed methods are introduced. In Section 4, the application of the proposed method in experimental data is explained. In Section 5, results and discussions are given. Section 6 concludes the paper.

\section{Signal Decomposition Method}

Empirical Mode Decomposition (EMD) is an effective method to decompose a nonlinear and nonstationary signal into a finite number of IMFs and a residue without leaving the time domain [7]. 
An IMF must satisfy the following two conditions:

1. The number of extrema and zero-crossings should be either equal or differ by one.

2. The mean value of the envelopes defined by local maxima and local minima is zero.

For a given signal $\mathrm{x}(\mathrm{t})$, the EMD algorithm can be described in the following steps:

Step 1: To extract all the local extrema (i.e., maxima and minima) from the signal.

Step 2: To obtain upper envelope $\mathrm{u}(\mathrm{t})$ and lower envelope $1(\mathrm{t})$ by connecting extrema using cubic splines.

Step 3: Determine the mean of splines $\left(m_{1}\right)$

$$
m_{1}=(u(t)+l(t)) / 2
$$

Step 4: To obtain a new signal $\left(c_{1}\right)$ by subtracting the mean $\left(m_{1}\right)$ from the original signal

$$
c_{1}=x(t)-m_{1}
$$

Step 5: Check if $c_{1}$ is an IMF

- If $c_{1}$ satisfies the EMD conditions, then save $c_{1}$ as the first IMF component else treat $c_{1}$ as the original signal.

Step 6: If $c_{1}$ is not IMF, then steps (1-5) are repeated for $\mathrm{k}^{\text {th }}$ times until $c_{1 k}$ becomes an IMF

$$
c_{1 k}=c_{1(k-1)}-m_{1 k}
$$

Where, $c_{1 k}$ is the first IMF of the signal and $m_{1 k}$ is the mean of the upper and lower envelope of $c_{1(k-1)}$.

Step 7: Calculate the residue 


$$
r_{1}=x-c_{1 k}
$$

Now, residue $r_{1}$ is considered as the original signal and repeats the procedure mentioned above to get the next IMF component. The above process is continued for $\mathrm{n}^{\text {th }}$ times until the final residue $r_{n}$ becomes monotonic function or constant, i.e., no further IMF can be extracted from the residue signal.

Finally, the signal $\mathrm{x}(\mathrm{t})$ can be expressed in terms of IMFs and residue as follow:

$$
x(t)=\sum_{i=1}^{n} I M F_{i}+r_{n}
$$

Where, $\mathrm{n}$ is the number of IMF components.

However, EMD includes mode aliasing and over decomposition problems. In order to overcome this, CEEMD is used.

In this method, two sets of white Gaussian noise are added to the original signal with opposite signs. CEEMD can remove the residual noise in the IMFs and improves computational efficiency. The CEEMD process is described in the following steps:

1. The white Gaussian noise with opposite signs is added to the original signal $\mathrm{x}(\mathrm{t})$. The obtained two signals are:

$$
\left\{\begin{array}{l}
x^{+}{ }_{j}=x(t)+w_{j}(t) \\
x^{+}{ }_{j}=x(t)-w_{j}(t)
\end{array}, \mathrm{j}=1,2,3, \ldots, \mathrm{n}\right.
$$

Where, $w_{j}(t)$ is the white gaussian noise with $\mathrm{j}^{\text {th }}$ noise realization.

2. The two signals such as $x^{+}{ }_{1}$ and $x^{-}$are decomposed by EMD separately, and two sets of components $I M F_{+1 i}, I M F_{-1 i}$ are achieved.

3. The above steps are repeated for $\mathrm{n}^{\text {th }}$ times, and $\mathrm{n}$ sets of $I M F_{+j i} \& I M F_{-j i}$ are obtained. 
4. Finally, the decomposition results are obtained by taking averaging operation for $\mathrm{n}$ sets of $I M F_{+j i} \& I M F_{-j i}$ components.

$$
I M F_{i}=\frac{1}{2 n} \sum_{j=1}^{n}\left(I M F_{+j i}+I M F_{-j i}\right)
$$

\section{The Proposed Denoising Method}

Recently, various works are conducted for bearing fault diagnosis by realizing the problem of IMF selection and the choice of thresholding operation for denoising the signals. The proposed method allows selecting a noise-predominant IMFs and low-frequency informative IMFs that carry the maximum fault information. The selected noise predominant modes are subjected to an adaptive thresholding method to remove excessive noise. Finally, signals are reconstructed using denoised IMFs and low-frequency IMFs. A flowchart of CEEMD-ATD (proposed method) for early fault detection in the bearing is described in Figure 1.

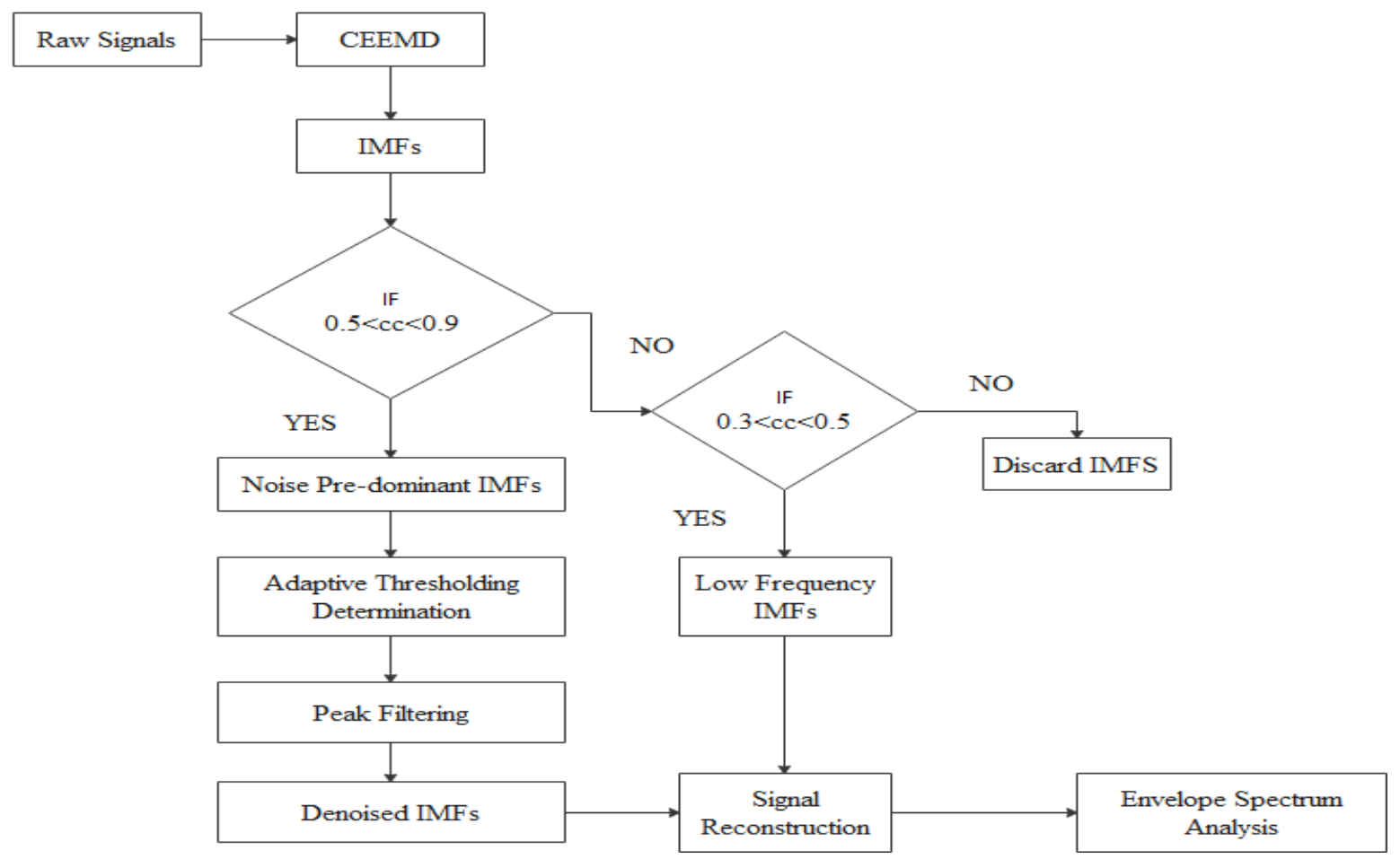

Figure 1: Flow Chart of CEEMD-ATD (Proposed method) for Bearing Fault Diagnosis. 
Once the raw signals are decomposed by CEEMD and sets of IMFs are obtained. IMFs grouping and selection, thresholding operation, and signal reconstruction are carried out as explained in the subsequent points.

\section{IMFs Grouping}

CEEMD method decomposes the bearing signal into multiple IMFs and residuals. The selection of proper IMFs is necessary to extract useful information about the bearing fault. In this work, the correlation coefficient is used to select and classify IMFs.

For the given signal $x(n)$ and $y(n)$, the correlation coefficient is defined as

$$
\rho_{x y}=\frac{\sum_{n=1}^{N}(x(n)-\overline{\mathrm{x}})(y(n)-\bar{y})}{\sqrt{\sum_{n=1}^{N} x(n)-\overline{\mathrm{x}}} \sqrt{\sum_{n=1}^{N} y(n)-\bar{y}}}
$$

Where, $\overline{\mathrm{x}}$ and $\bar{y}$ is the mean of the signal $x(n)$ and $y(n)$, respectively. $\rho_{x y}$ is the correlation coefficient value between the two signals $\mathrm{x}$ and $\mathrm{y}$.

In this work, IMFs are classified into four groups are as noisy IMFs, noise predominant IMFs, low-frequency IMFs, and residuals, respectively, based on correlation coefficient value. Table 1 shows the correlation coefficient (CC) values and their interpretation. The CC of each IMF and the original signal are calculated. The value of $\mathrm{CC}$ in between 0.9 to 1 indicates that the IMFs are highly correlated to the original signal and can be considered as a noisy original signal, and it displays the same behavior as the original signal. So, extremely high positive correlated IMFs are discarded for denoising and signal reconstruction. IMFs having CC values in between 0.5 to 0.9 are considered as noise predominant IMFs, and values in between 0.3 to 0.5 are considered as lowfrequency IMFs and are considered for further processing. Whereas IMFs with CC value less than 0.3 are showing negligible correlation and carries no relevant information about bearing fault. Therefore, it is discarded for signal reconstruction. 
Table 1: Interpretation of correlation coefficient value

\begin{tabular}{ccc}
\hline $\begin{array}{c}\text { Correlation } \\
\text { coefficient Value }\end{array}$ & Normal Interpretation & $\begin{array}{c}\text { Interpretation in terms of Bearing } \\
\text { IMFs }\end{array}$ \\
\hline $\mathbf{0 . 9 0}$ to 1.00 & Extremely high positive correlation & Noisy IMFs \\
\hline $\mathbf{0 . 7 0}$ to 0.90 & High positive correlation & Noise Pre-Dominant IMFs \\
\hline $\mathbf{0 . 5 0}$ to 0.70 & Moderate positive correlation & Low-frequency IMFs \\
\hline $\mathbf{0 . 3 0}$ to 0.50 & Low positive correlation & Residual (Discard) \\
\hline $\mathbf{0 . 0 0}$ to 0.30 & negligible correlation & \\
\hline
\end{tabular}

\section{Adaptive Thresholding Method based on the 3 $\sigma$ Criterion}

IMF with the highest kurtosis value contains useful information about fault, but they are generally contaminated with excessive noise, and the noises are present in the signal in smaller values. Therefore, in the signal denoising process, the first step is to establish a threshold value between the useful information and the noises in the signals. In this work, the threshold value is set for bearing signals based on the $3 \sigma$ criterion. The adaptive threshold determination method procedure is described in the following steps:

Step1: Calculate the standard deviation of the $\operatorname{IMF}\left(\sigma_{o}\right)$ :

$$
\sigma_{o}=\sqrt{\frac{1}{K-1} \sum_{k=1}^{K}(I M F(k)-\overline{I M F(k)})^{2}}
$$

Where, $\mathrm{K}$ is the length of the IMF and $\overline{\operatorname{IMF}(k)}$ is the mean of the $\operatorname{IMF}(\mathrm{k})$.

Step2: Remove the value that is greater than $3 \sigma_{o}$ from the absolute $\operatorname{IMF}(k)-\overline{\operatorname{IMF}(k)}$. Now, the remaining values are treated as a new noisy signal $\operatorname{IMF}^{1}(k)$. 
Step3: Calculate the standard deviation for the new noisy signal $I M F^{1}(k)$ represented by $\sigma_{1}$.

$$
\sigma_{1}=\sqrt{\frac{1}{K_{1}-1} \sum_{k=1}^{K_{1}}\left(I M F^{1}(k)-\overline{I M F^{1}(k)}\right)^{2}}
$$

Where, $K_{1}$ is the length of the remaining values, i.e., new noisy signal $I M F^{1}(k)$.

Step 4: In general, the absolute value in $I M F^{i-1}(k)-\overline{I M F^{l-1}(k)}$ remove the values which are greater than $3 \sigma_{i-1}$. The remaining values are treated as a new noisy signal $I M F^{i}(k)$ of length $K_{i}$, which is the number of remaining values. Now, the standard deviation $\left(\sigma_{i}\right)$ for new noisy signal $I M F^{i}(k)$ is given by

$$
\sigma_{i}=\sqrt{\frac{1}{K_{i}-1} \sum_{k=1}^{K_{i}}\left(I M F^{i}(k)-\overline{I M F^{\imath}(k)}\right)^{2}}
$$

Step 5: Steps 1-3 are repeated until there is no value in absolute $I M F^{i}(k)-\overline{I M F^{\iota}(k)}$ greater than $3 \sigma_{i}$. Now, $3 \sigma_{i}$ is determined as the final threshold value.

$$
\lambda=3 \sigma_{i}
$$

Where, $\lambda$ is the final threshold value.

In this method, at every phase of iteration, calculated $3 \sigma$ decreases gradually, and much "pure Noise" will be screened out. Therefore, the last $3 \sigma_{i}$ represents the final threshold. This threshold value will be used in the peak filtering denoising method. 


\section{Peak Filtering Denoising Method}

In hard and soft thresholding, if any value in the signal is smaller than the threshold, it is considered noise and set to zero. However, some of the values smaller than the threshold belongs to the useful parts of the signal, and it should be retained to prevent the loss of local information about bearing fault. The common methods for denoising of non-stationary signals are wavelet transform and EMD. In the wavelet transform, the appropriate selection of mother wavelet and decomposition level are essential parameters for denoising the non-stationary signal. However, the selection of these two parameters for the denoising process is a difficult task. In this work, the peak filtering technique is used to ease the thresholding operation and retain maximum information from denoised signals. The peak filtering method working is explained in the form of a flow chart as shown in figure 2.

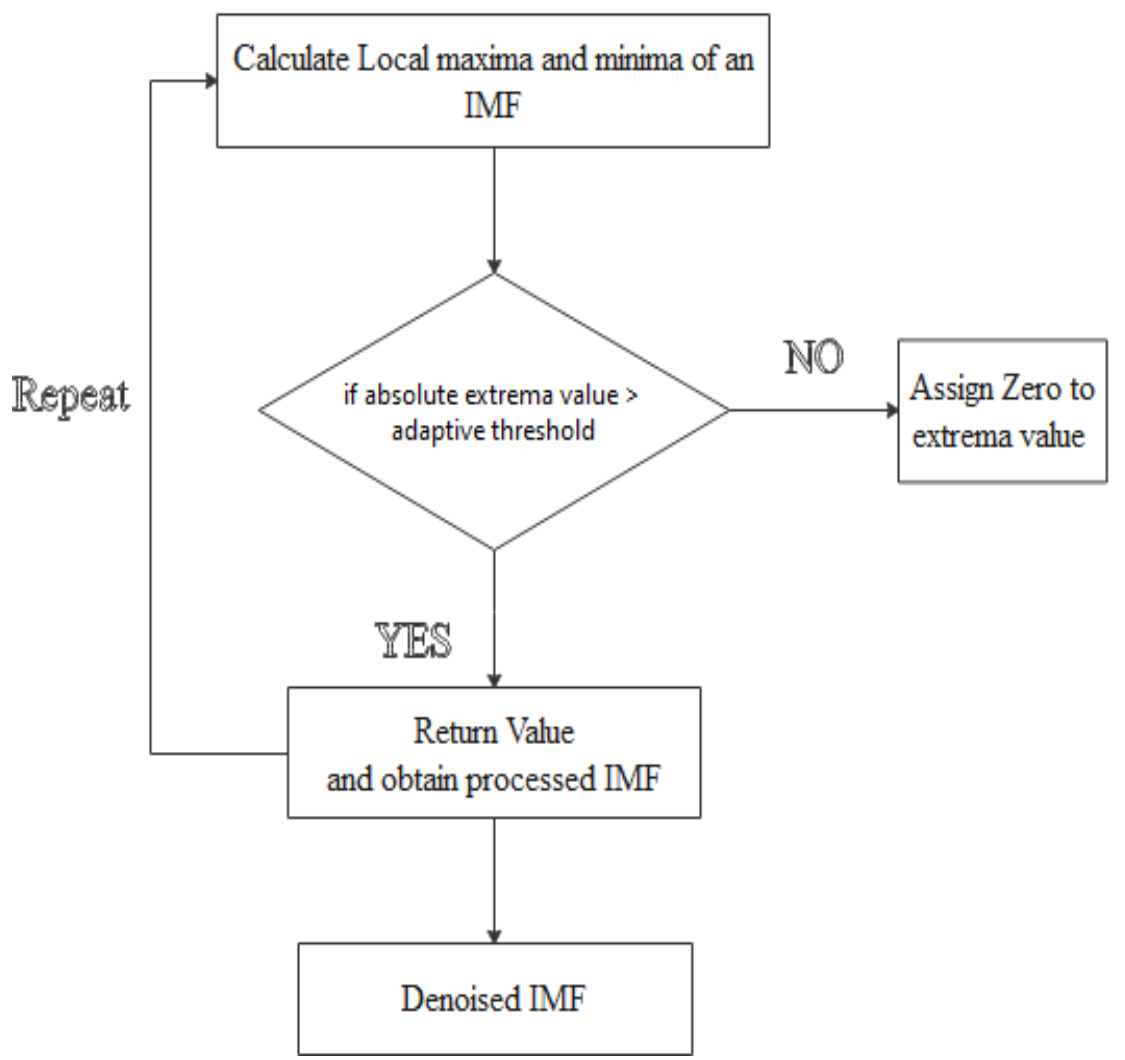

Figure 2: Flow chart shows the working of the peak filtering denoising Method. 
Noise predominant IMFs are subjected to peaking filtering denoising methods. In this method, local maxima and minima are calculated for each noise predominant IMFs. The absolute values of the local maxima and minima, i.e., extrema values, are compared with the adaptive threshold value $\lambda$ determined by the $3 \sigma$ criterion method. The extrema, whose absolute values are smaller than the threshold are assigned zero, and values above the threshold are returned as it is, and the processed IMF is obtained. The signal's length remains the same as of original signal to prevent the aliasing problem. This process is repeated until there is no more extrema value remaining in the signal to be set to zero. Finally, the remaining processed IMF is considered a denoised IMF. Therefore, filtering the peaks repeatedly, which is smaller than the threshold value, reduces the noise in bearing signals and retains the maximum fault information.

\section{Application to Experimental Data}

The proposed method performance is tested on a bearing dataset provided by Case Western Reverse University (CWRU) for fault detection and identification. The test facility layout by CWRU is shown in Figure 3 [24]. It consists of a two HP electric motor and dynamometer configuration connected with a shaft. The torque transducer and encoder are mounted in the shaft. The Load is being applied to the motor with the help of a dynamometer.

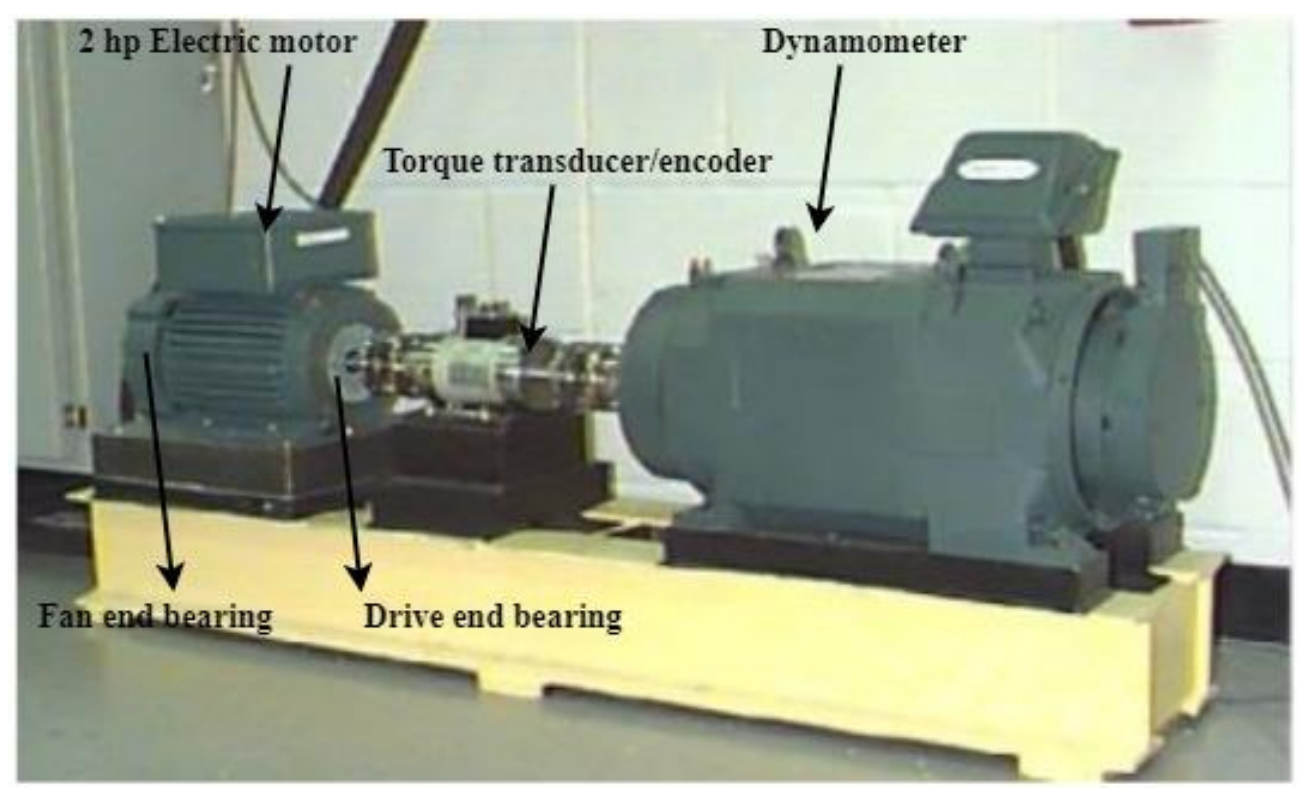

Figure 3: CWRU bearing test rig [24] 
Table 2: Geometrical characteristics of ball bearing

\begin{tabular}{cc}
\hline Parameter & Value \\
\hline Inside diameter & $25 \mathrm{~mm}$ \\
\hline outside diameter & $52 \mathrm{~mm}$ \\
\hline Thickness & $15 \mathrm{~mm}$ \\
\hline Pitch diameter & $39 \mathrm{~mm}$ \\
\hline Ball diameter & $8 \mathrm{~mm}$ \\
\hline Number of Rolling bodies & 9 \\
\hline Contact angle & $0 \mathrm{rad}$ \\
\hline
\end{tabular}

The vibration signals of ball bearings used in this article are of type 6205-2RS JEM SKF, and its geometrical specifications are given in Table 2. For the test, a fault diameter of $0.1778 \mathrm{~mm}$ on drive end bearing is selected in this paper. The faults are artificially seeded on the motor's drive end bearing by using electro-discharge machining (EDM). In this test facility, different sets of faulty bearings are used that are reinstalled to the motor for testing, and the vibration signals are measured. The motor bearing is tested for four different speeds of 1797, 1772, 1750, and 1730 rpm, respectively, with a sampling frequency of $48000 \mathrm{~Hz}$ is chosen in this paper.

The rolling element bearing with defect produces a cycle of impacts, which is repeated periodically at a rate known as the bearing frequencies. Each bearing component exhibits a unique defect frequency that depends on the geometric characteristics of the bearing. In this work, the vibration signals are corresponding to the bearing with inner race fault (IRF), outer race fault (ORF), and healthy bearing (HB).

BPFO (Ball Pass Frequency Outer) corresponds to the number of balls passing through a crack present in the outer race each time when the shaft is making a complete rotation. The characteristic frequency for ORF is calculated by using the following equation

$$
B P F O, F_{o}=\frac{n}{2} \times f_{s} \times\left(1-\frac{d}{D} \cos \varphi\right)
$$


BPFI (Ball Pass Frequency Inner) generally corresponds to the number of balls passing through a crack present in the inner race track each time the shaft is making a complete rotation. The characteristic frequency for IRF is calculated by using the following equation

$$
B P F I, F_{i}=\frac{n}{2} \times f_{s} \times\left(1+\frac{d}{D} \cos \varphi\right)
$$

Where, $f_{s}$ is the rotational frequency of the shaft in $\mathrm{rad} / \mathrm{s}, \mathrm{d}$ is the diameter of rolling bodies, $\mathrm{D}$ is the pitch diameter, $\mathrm{n}$ is the number of rolling bodies, and $\varphi$ is the contact angle. By substituting the parameter values of the bearing as given in table 2 in equation 4.1 and 4.2, respectively, the fault frequencies such as $F_{o}$ and $F_{i}$ are calculated at different speeds are presented in Table 3.

Table 3: Calculated Fault Frequencies

\begin{tabular}{|c|c|c|}
\hline Motor speed (rpm) & BPFI, $F_{i}(\mathrm{~Hz})$ & $\mathrm{BPFO}, F_{o}(\mathrm{~Hz})$ \\
\hline 1797 & 162.44 & 107.10 \\
\hline 1772 & 160.18 & 105.61 \\
\hline 1750 & 158.20 & 104.30 \\
\hline 1730 & 156.36 & 102.93 \\
\hline
\end{tabular}

\section{Results and Discussion}

This section discusses the result of CEEMD +ATD (Proposed Method) as applied to CRWU bearing dataset for early fault diagnosis. To validate the effectiveness of the proposed method, the result of the denoised signal is compared with the two other conventional methods based on kurtosis value.

The bearing signals with IRF, ORF, and HB for motor speed running at $1797 \mathrm{rpm}$ are shown in figure 4. In this work, CEEMD is used for a different set of Gaussian white noise (refer to equation 2.7) for bearing signals decomposition. The result of the first 8 IMFs of decomposed signal for IRF, ORF, and HB running at $1797 \mathrm{rpm}$ are shown in figures 5, 6, and 7. 

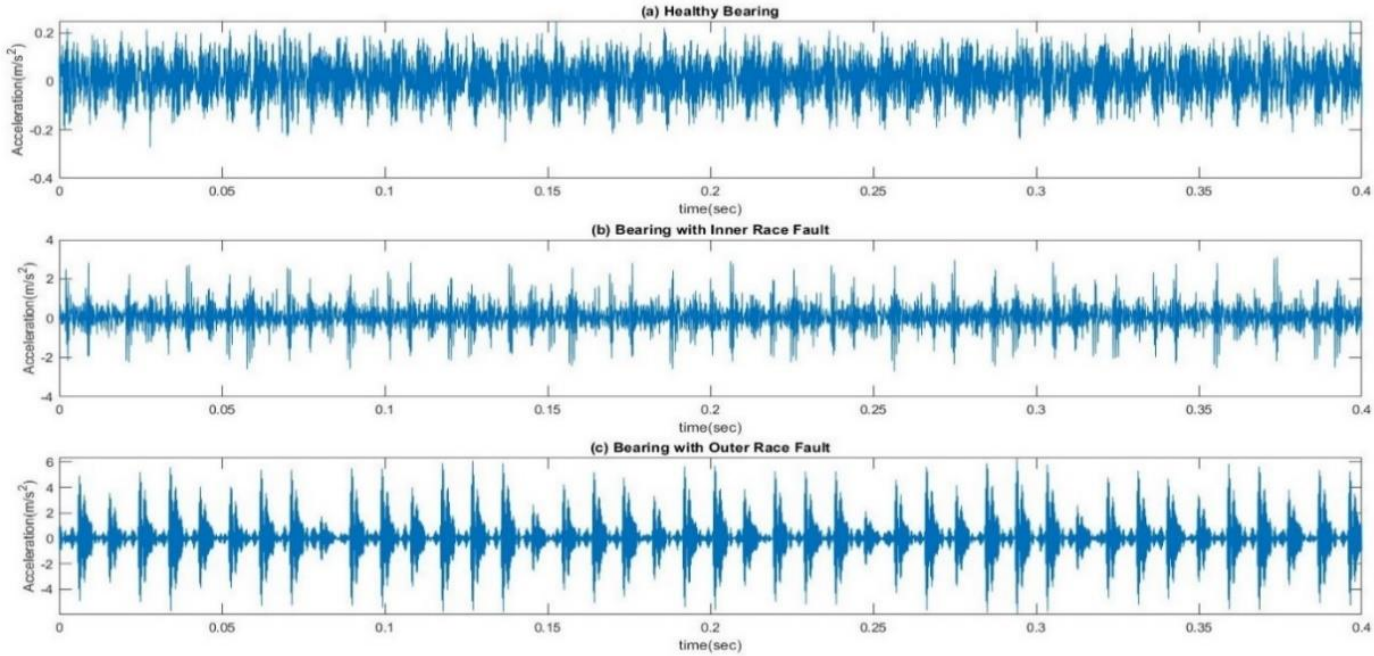

Figure 4: Original Signals (a) Healthy Bearing (b) Bearing with Inner Race Fault and (c) Bearing with Outer Race Fault
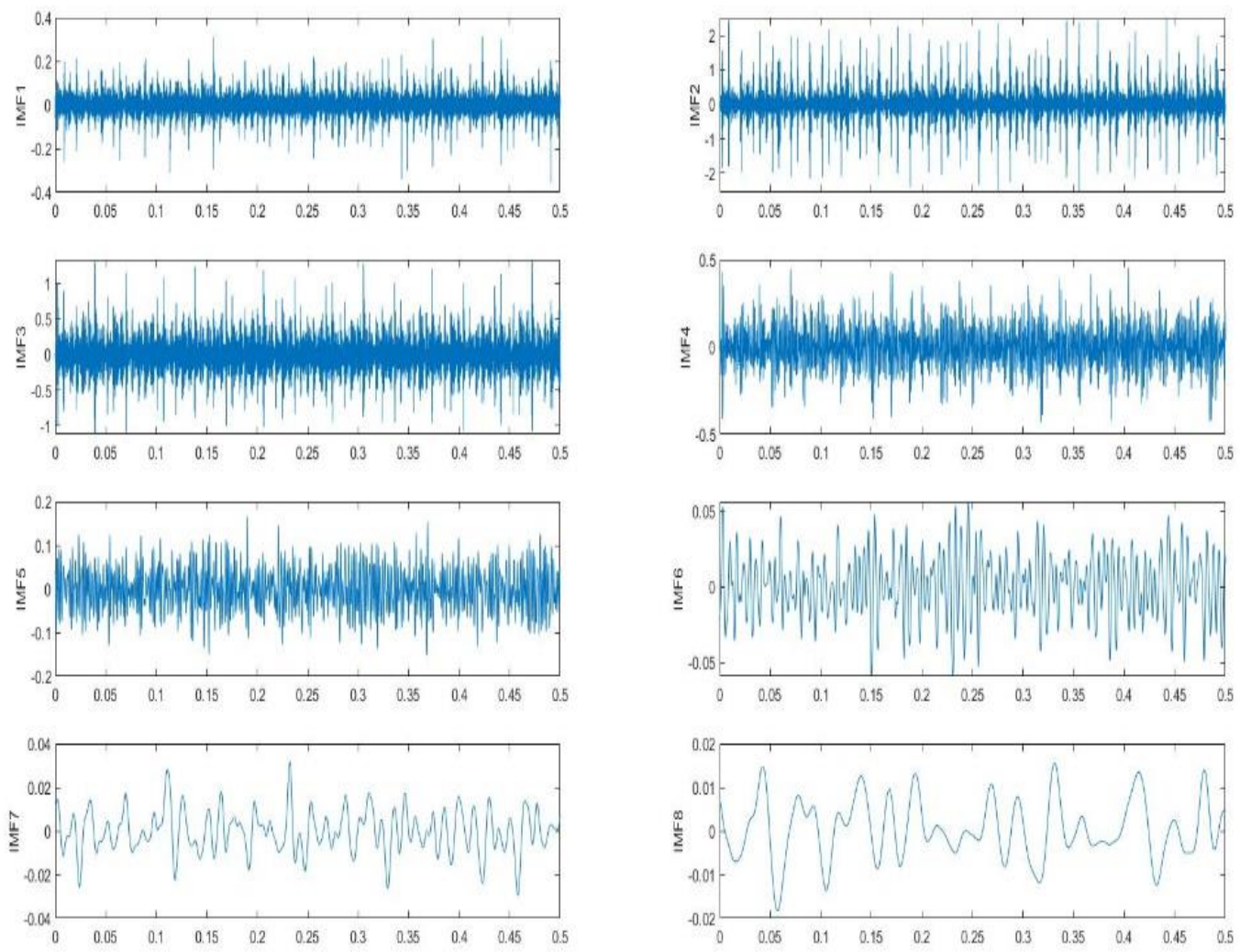

Figure 5: Inner race fault signal decomposed by CEEMD 

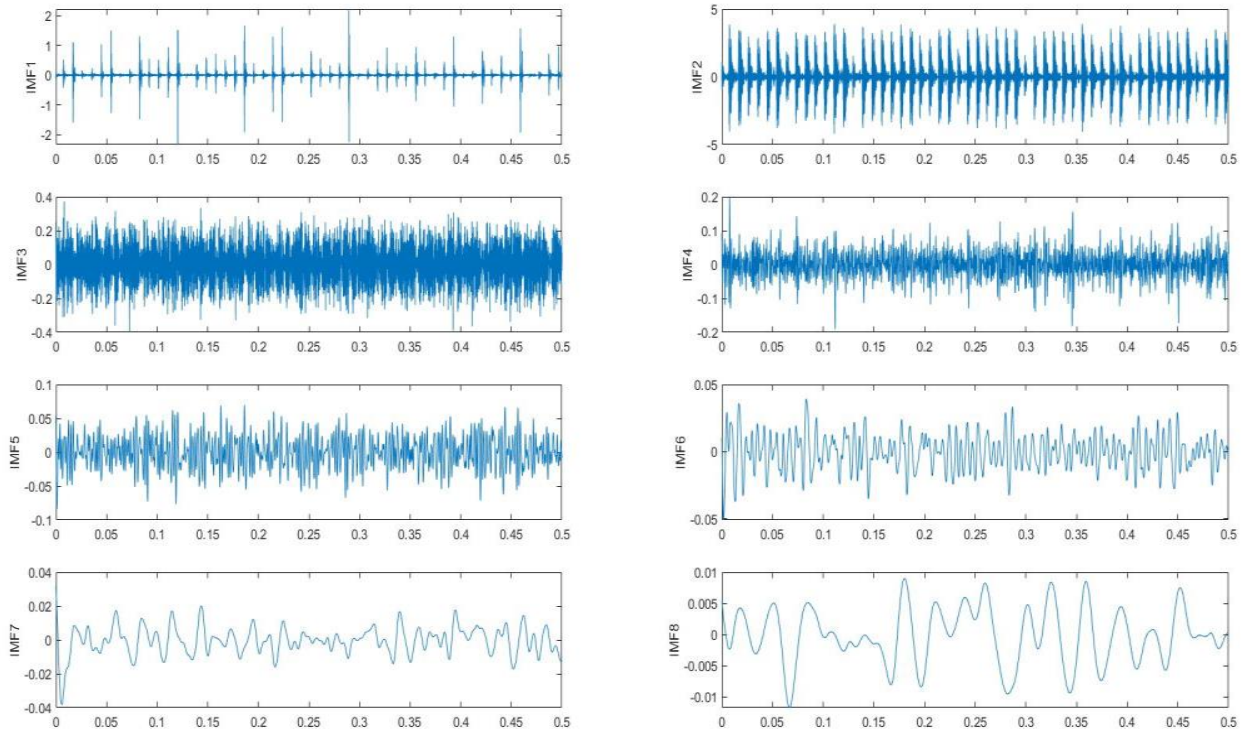

Figure 6: Outer race fault signal decomposed by CEEMD
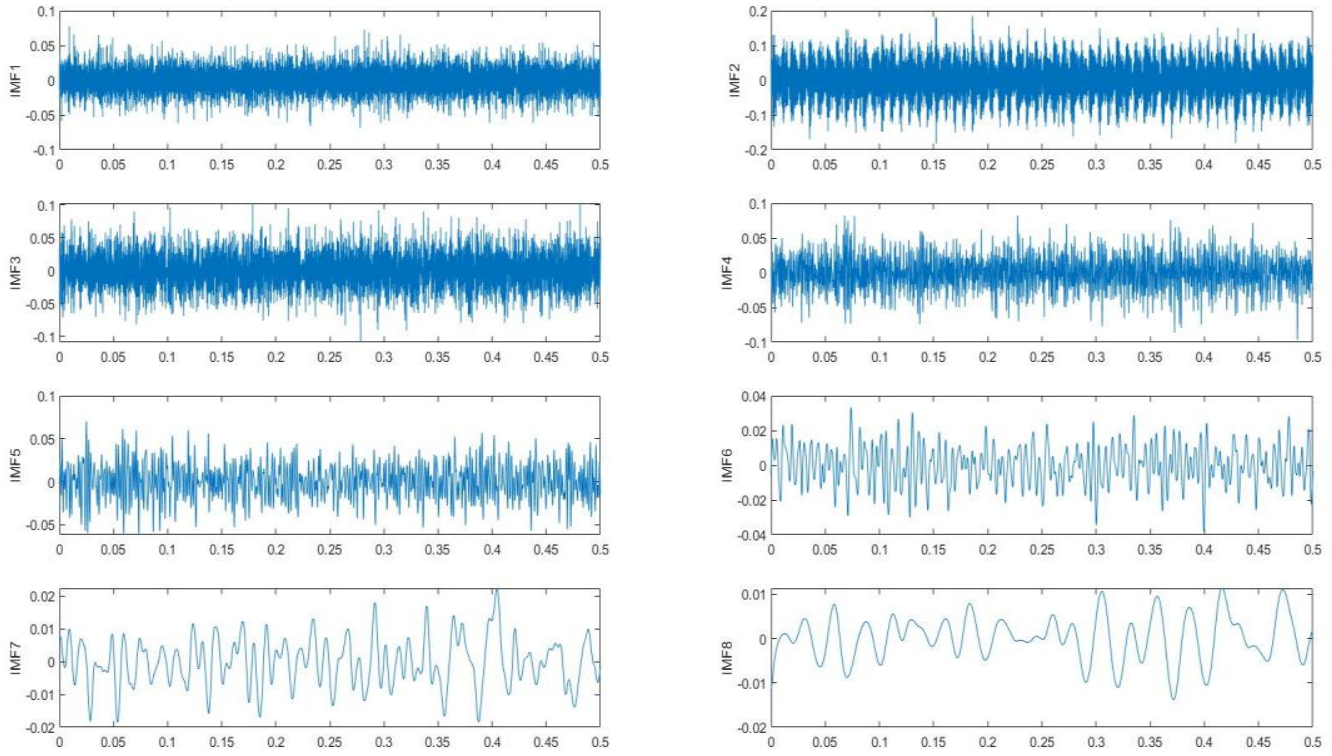

Figure 7: Healthy bearing signal decomposed by CEEMD

The CC is calculated for each IMFs with respect to the original signal for IRF, ORF, and HB, respectively, as mentioned in Tables 4, 5, and 6, respectively. The signal with CC value above 0.9 coincides with the original signal and is treated as a noisy original signal and consideration of such 
IMFs create redundancy as shown in Figures 8 and 9. So, too strong IMFs are discarded for signal reconstruction.

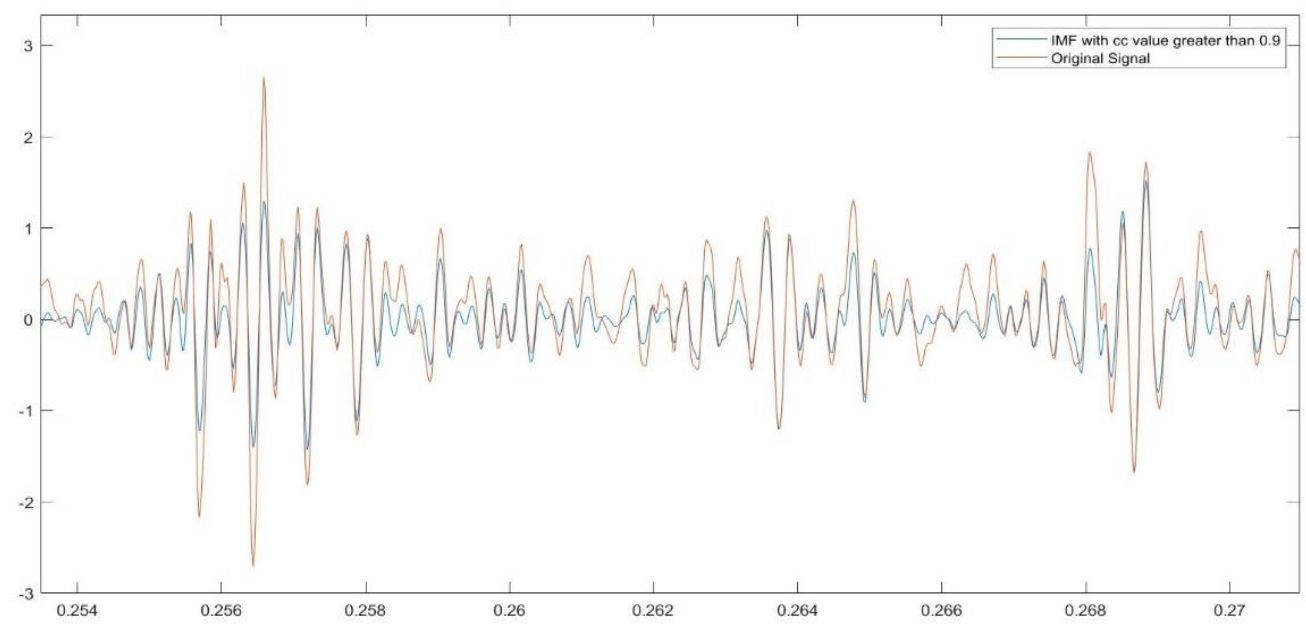

Figure 8: IMF vs Original signal for Inner race fault

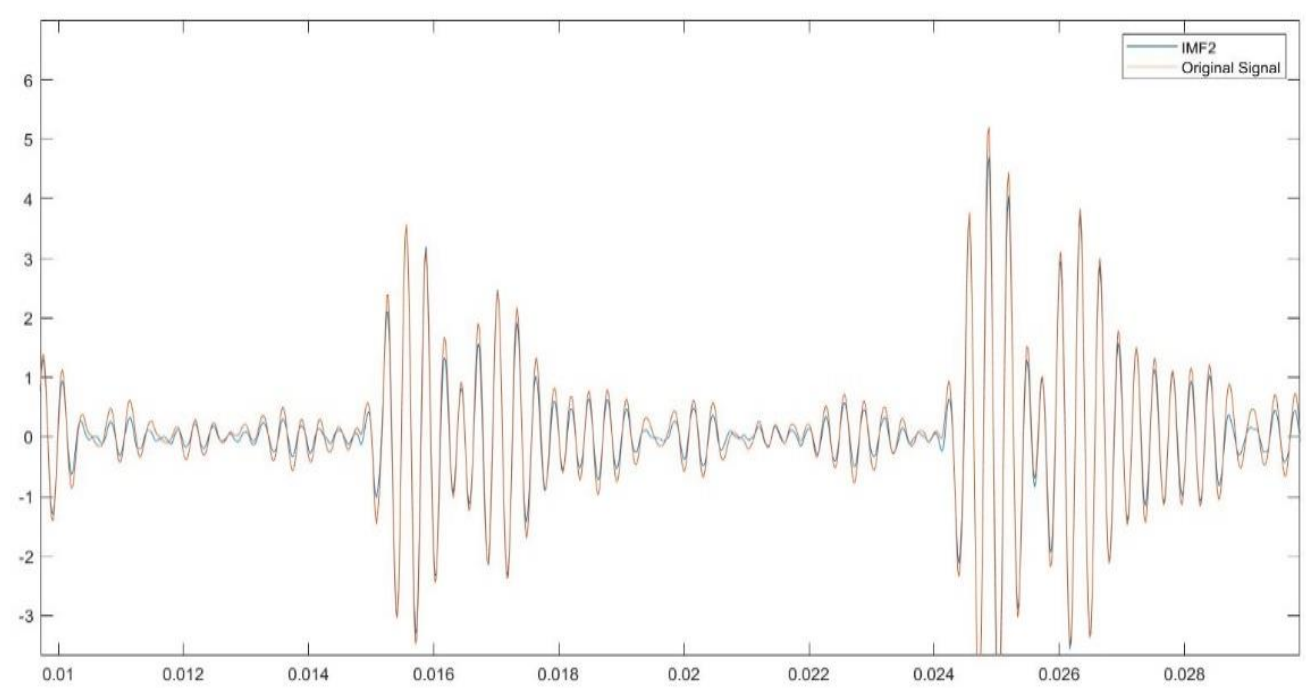

Figure 9: IMF2 vs Original signal for outer race fault

In the case of IRF, based on CC values as shown in table 4, IMF1 and IMF3 are selected as noise-predominant IMFs for adaptive thresholding and signal reconstruction for motor running at 1797 and 1772 rpm, respectively. However, IMF1, IMF2, and IMF3 are selected as noise- 
predominant IMFs for adaptive thresholding and signal reconstruction for motor speed running at 1750 and $1730 \mathrm{rpm}$, respectively.

Table 4: Correlation coefficient of inner race fault signal IMFs and the original signal rotating at 4 different speed

\section{Inner Race Fault}

\begin{tabular}{ccccccccc}
\hline & \multicolumn{7}{c}{ Correlation coefficient } \\
\cline { 2 - 9 } Motor speed & IMF1 & IMF2 & IMF3 & IMF4 & IMF5 & IMF6 & IMF7 & IMF8 \\
\hline $\mathbf{1 7 9 7}$ & 0.5955 & 0.9018 & 0.5339 & 0.2606 & 0.1140 & 0.0273 & 0.0016 & -0.0001 \\
\hline $\mathbf{1 7 7 2}$ & 0.5346 & 0.9040 & 0.6230 & 0.2784 & 0.1561 & 0.0299 & 0.0041 & 0.0004 \\
\hline $\mathbf{1 7 5 0}$ & 0.5219 & 0.8902 & 0.6315 & 0.2812 & 0.1686 & 0.0344 & 0.0051 & 0.0004 \\
\hline $\mathbf{1 7 3 0}$ & 0.5356 & 0.8646 & 0.6259 & 0.2989 & 0.1781 & 0.0285 & 0.0034 & -0.0003 \\
\hline
\end{tabular}

In the ORF case, the CC value of IMF2 is larger than 0.9, as shown in table 5. IMF3 to IMF8 having CC values less than 0.3 exhibits negligible correlation. Therefore, it should be removed. Finally, single noise predominant IMF1 is selected for adaptive thresholding and signal reconstruction for motor speed running at 1797, 1772, 1750, and $1730 \mathrm{rpm}$, respectively.

Table 5: Correlation coefficient of outer race fault signal IMFs and the original signal rotating at 4 different speed

\section{Outer Race Fault}

\begin{tabular}{ccccccccc}
\hline \multirow{2}{*}{ Motor speed } & \multicolumn{7}{c}{ Correlation coefficient } \\
\cline { 2 - 9 } & IMF1 & IMF2 & IMF3 & IMF4 & IMF5 & IMF6 & IMF7 & IMF8 \\
\hline $\mathbf{1 7 9 7}$ & 0.6712 & 0.9897 & 0.2965 & 0.0320 & 0.0198 & 0.0049 & 0.0005 & 0.0002 \\
\hline $\mathbf{1 7 7 2}$ & 0.6525 & 0.9920 & 0.2877 & 0.0324 & 0.0201 & 0.0039 & 0.0004 & -0.0001 \\
\hline $\mathbf{1 7 5 0}$ & 0.6747 & 0.9929 & 0.2811 & 0.0322 & 0.0167 & 0.0068 & 0.0005 & 0.0003 \\
\hline $\mathbf{1 7 3 0}$ & 0.6559 & 0.9909 & 0.2978 & 0.0398 & 0.0189 & 0.0077 & 0.0003 & -0.0001 \\
\hline
\end{tabular}

In the case of $\mathrm{HB}$, based on CC values as shown in table 6, IMF2 and IMF3 are selected as noise-predominant IMFs for adaptive thresholding, and IMF1, IMF4, and IMF 5 are selected as low-frequency informative IMFs for signal reconstruction for motor running at $1797 \mathrm{rpm}$. IMF2 
is selected as noise-predominant IMFs for adaptive thresholding, and IMF1, IMF3, IMF4, and IMF 5 are chosen as low-frequency informative IMFs for signal reconstruction for motor running at 1772,1750 , and $1730 \mathrm{rpm}$.

Table 6: Correlation coefficient of healthy bearing signal IMFs and the original signal rotating at 4 different speed

\begin{tabular}{ccccccccc}
\hline & \multicolumn{7}{c}{ Healthy Bearing } \\
\hline \multirow{2}{*}{ Motor speed } & \multicolumn{7}{c}{ Correlation coefficient } \\
\cline { 2 - 9 } & IMF1 & IMF2 & IMF3 & IMF4 & IMF5 & IMF6 & IMF7 & IMF8 \\
\hline $\mathbf{1 7 9 7}$ & 0.3480 & 0.8016 & 0.5209 & 0.3376 & 0.3113 & 0.1200 & 0.0091 & 0.0008 \\
\hline $\mathbf{1 7 7 2}$ & 0.3768 & 0.6574 & 0.4193 & 0.3903 & 0.4880 & 0.1606 & 0.0071 & 0.0005 \\
\hline $\mathbf{1 7 5 0}$ & 0.4532 & 0.6529 & 0.3922 & 0.3865 & 0.4493 & 0.1657 & 0.0120 & 0.0008 \\
\hline $\mathbf{1 7 3 0}$ & 0.4775 & 0.6543 & 0.4290 & 0.3995 & 0.4133 & 0.1471 & 0.0093 & 0.0012 \\
\hline
\end{tabular}

Once, the denoised signals are obtained for bearing with IRF, ORF, and HB. The kurtosis and envelope spectrum analysis is performed on the denoised signal, as explained in the subsequent paragraphs considering the three cases such as IRF, ORF, and HB.

\section{Case1: Bearing Signal with Inner Race Fault (IRF)}

The bearing signals with IRF for motor speed running at four speeds of 1797, 1772, 1750, and 1730 rpm, respectively, are decomposed by CEEMD and ATD. The signals denoised by proposed methods are presented in figure 10. Figure 11 shows the comparison between WTD and ATD for inner race fault signal. WTD result shows better continuity of signal but missed some of the fault impulses which may lead to loss of information. However, the proposed ATD captured all the fault impulses and can extract maximum fault information.

The kurtosis values are calculated for denoised signals by the proposed method and compared with the two other conventional methods, as shown in figure 12. The original signals kurtosis values are 6.01, 7.40, 7.39, and 6.95 at rotating speeds of 1797, 1772, 1750, and $1730 \mathrm{rpm}$. The denoised signals kurtosis values by conventional method-1 [9] are 5.41, 16.79, 16.46, and 14.62 whereas, conventional method-2 [12] are 89.06, 65.60, 70.99, and 60.99 at rotating speeds of 1797 , 1772, 1750, and $1730 \mathrm{rpm}$, respectively. The kurtosis values for the proposed method denoised signals are $171.33,127.90,73.94$, and 61.75 at rotating speeds $1797,1772,1750$, and $1730 \mathrm{rpm}$, 
respectively. It is clearly observed from the kurtosis value comparison, as shown in figure 12 , that the proposed method shows significant improvement in the sensitivity of the kurtosis parameter to identify the fault in an early way as compared to the two other conventional methods. The proposed methods denoised signal is then subjected to envelope spectrum analysis running at four different speeds, as shown in figure 13. The results obtained by envelope spectrum analysis indicates that the peaks and their harmonics appear at the shaft rotational speed (Fr) and BPFI (Fi). The peak frequencies by the proposed method are equal to the calculated values presented in table 3 . The existence of peak at BPFI (Fi) and its harmonics reveals that there is a defect present in the inner race of the bearing.
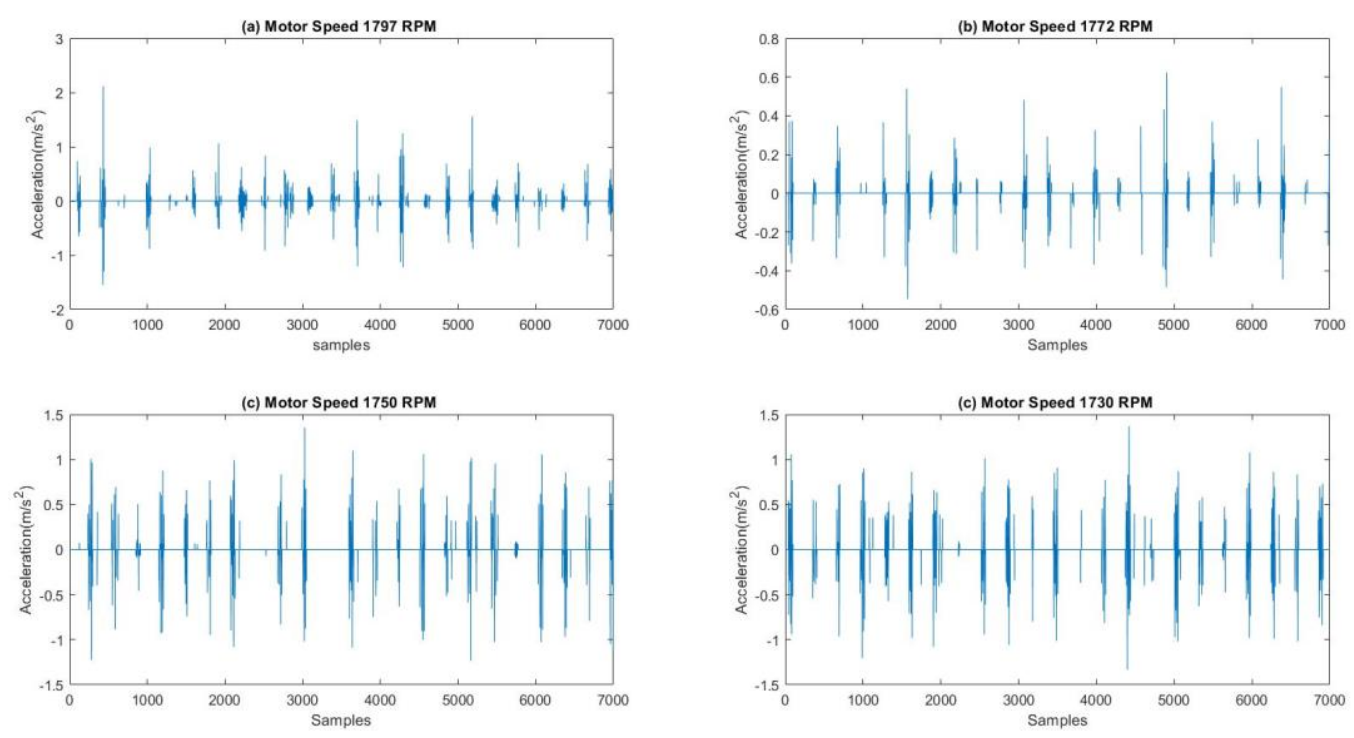

Figure 10: Denoised signals of bearing with inner race fault running at 4 different speed by the proposed method 


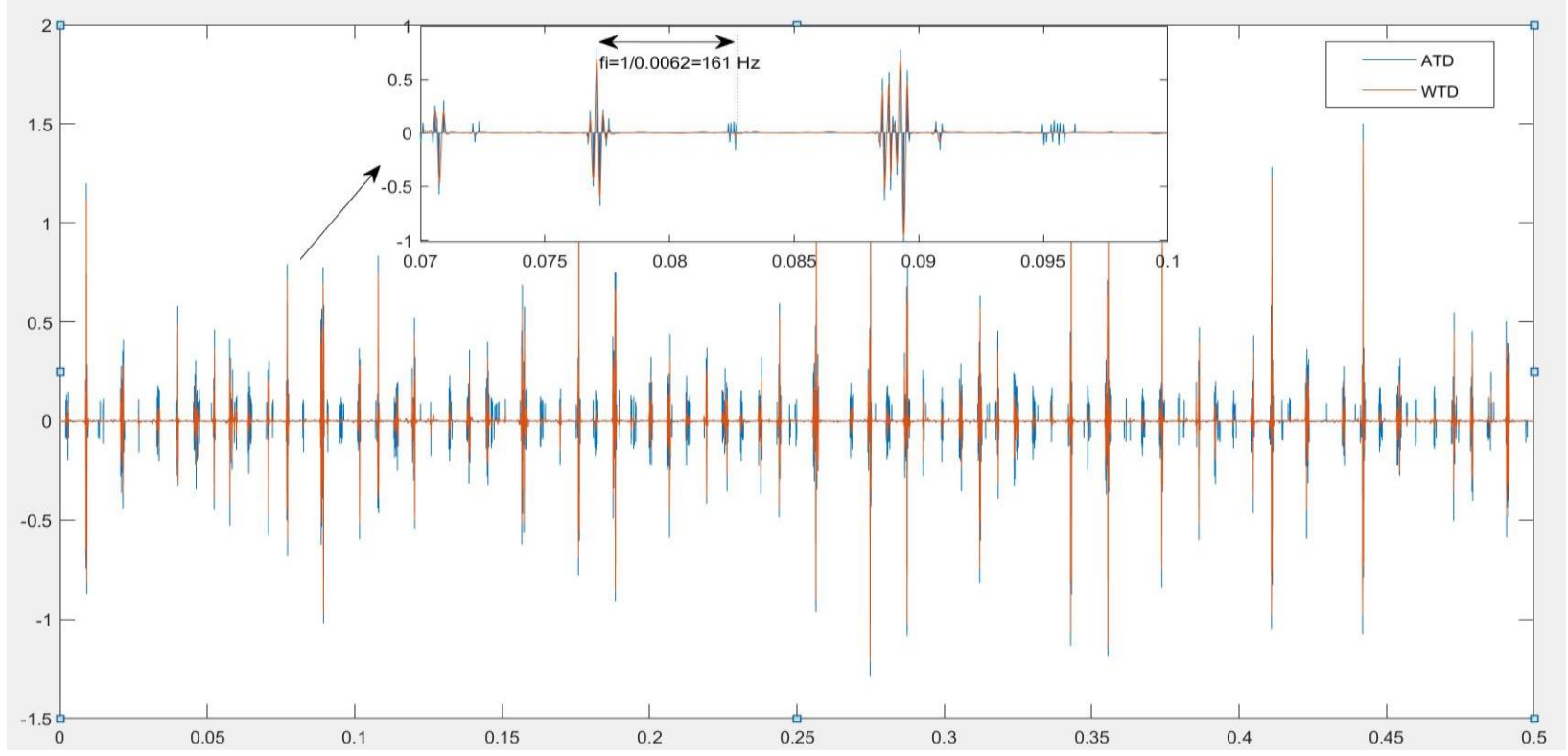

Figure 11: Inner Race fault signal denoised by WTD and ATD.

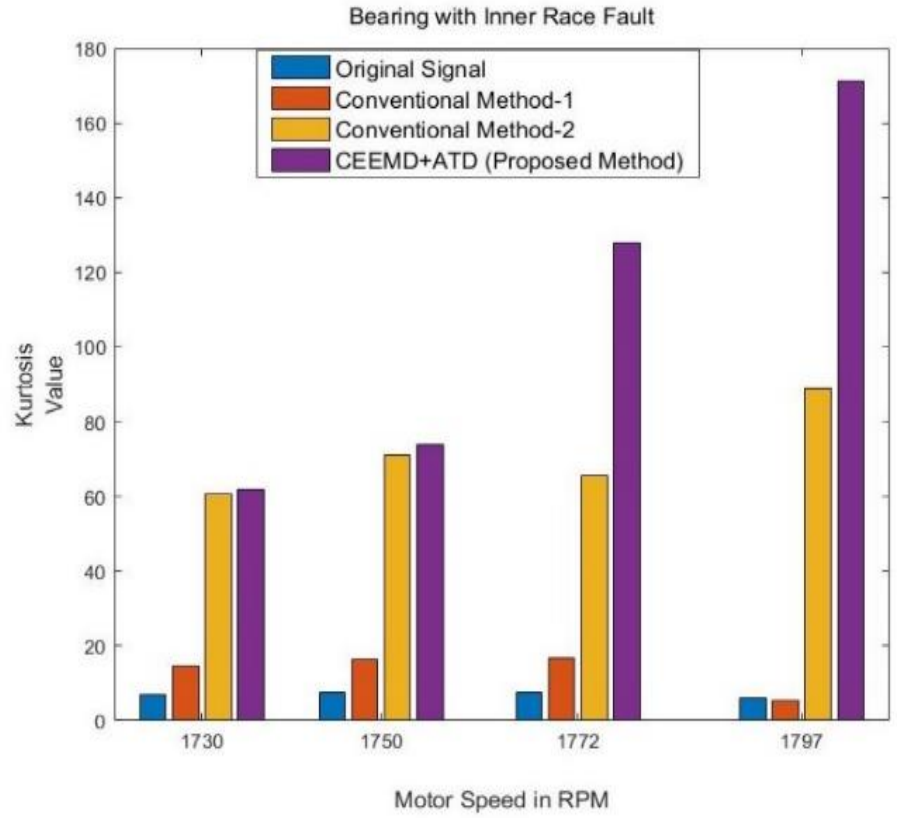

Figure 12: Kurtosis value comparison for Inner race fault 

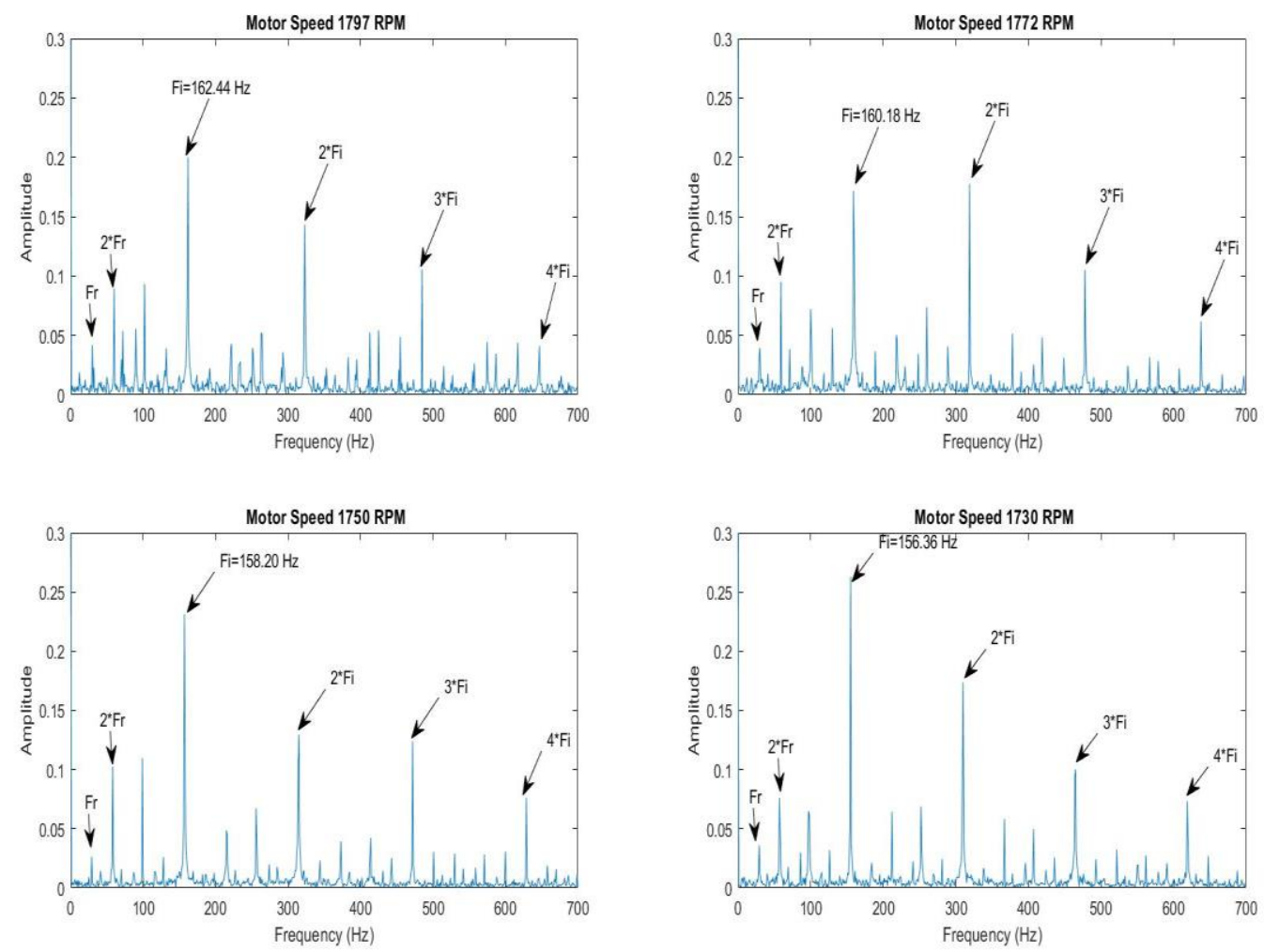

Figure 13: Envelope Spectra analysis of bearing with inner race fault signal by the proposed method.

\section{Case2: Bearing Signal with Outer Race Fault (ORF)}

CEEMD and ATD decompose the bearing signals with ORF for motor running at four speeds 1797, 1750, 1772, and $1730 \mathrm{rpm}$. The ORF signals denoised by the proposed method are shown in figure 14. Figure 15 shows the comparison between WTD and ATD for outer race fault signal. WTD result shows better continuity of signal but missed some of the fault impulses which may lead to loss of information. However, the proposed ATD captured all the fault impulses and can extract maximum fault information.

The kurtosis values are calculated for denoised signals by the proposed method and compared with the two other conventional methods, as shown in figure 16. The original signals kurtosis values are 6.80, 6.90, 7.009, and 7.25 at rotating speeds of 1797, 1772, 1750, and $1730 \mathrm{rpm}$. The denoised signals kurtosis values by conventional method-1 [9] are 48.37, 48.90, 44.20 and 43.78 whereas, for conventional method-2 [12] are 67.69, 86.96, 65.39 and 70.97 at rotating speeds 1797, 1772, 1750, and $1730 \mathrm{rpm}$, respectively. The kurtosis values for the proposed method denoised 
signals are 276.46, 242.33, 213.70, and 311.10 at rotating speeds of $1797,1772,1750$, and 1730 rpm, respectively. It is clearly observed from the kurtosis value, as shown in figure 16, that the proposed method shows significant improvement in the sensitivity of the kurtosis parameter to identify the fault in an early way as compared to the two other conventional methods. The proposed method denoised signals are then subjected to envelope spectrum analysis running at four different speeds, as indicated in figure 17. The envelope spectrum analysis results indicate that the peaks and their harmonics appear at the shaft rotational speed (Fr) and BPFO (Fo). The peak frequencies by the proposed method are equal to the calculated values presented in table 3 . The existence of peaks at BPFO (Fo) and its harmonics reveals that there is a defect present in the outer race of the bearing.
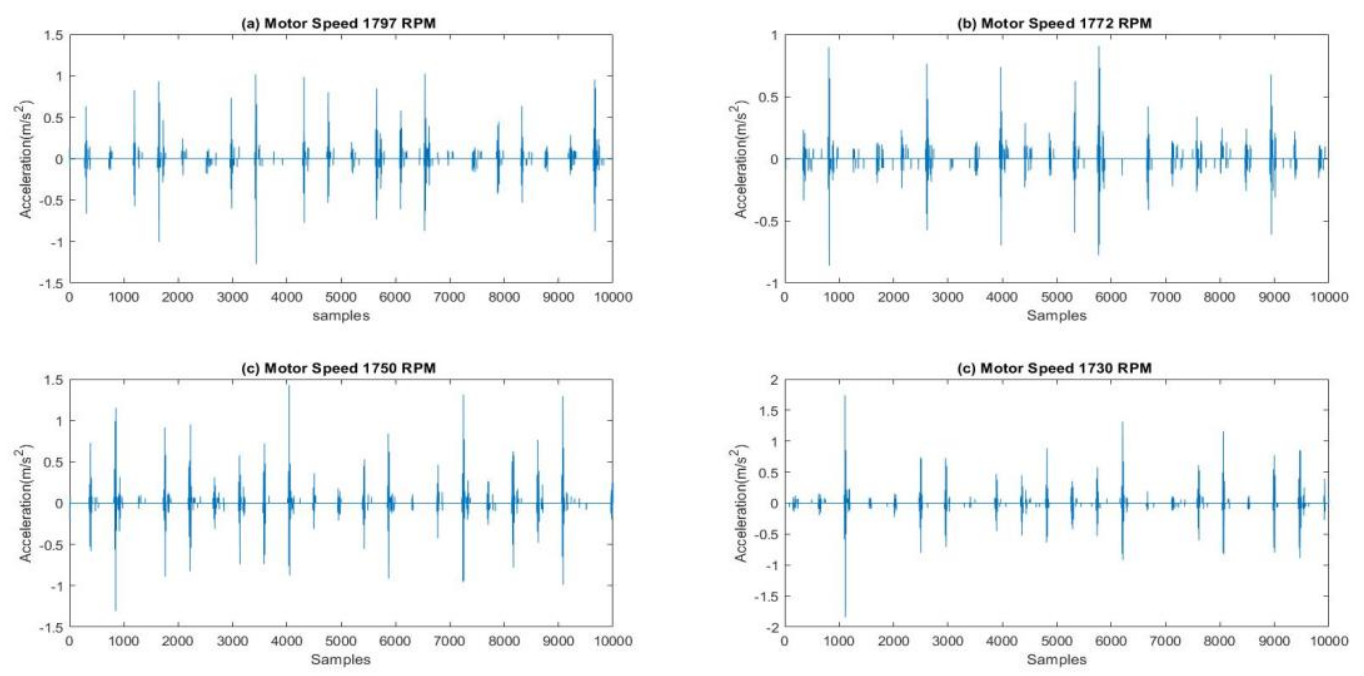

Figure 14: Denoised signals of bearing with outer race fault running at 4 different speed by the proposed method 


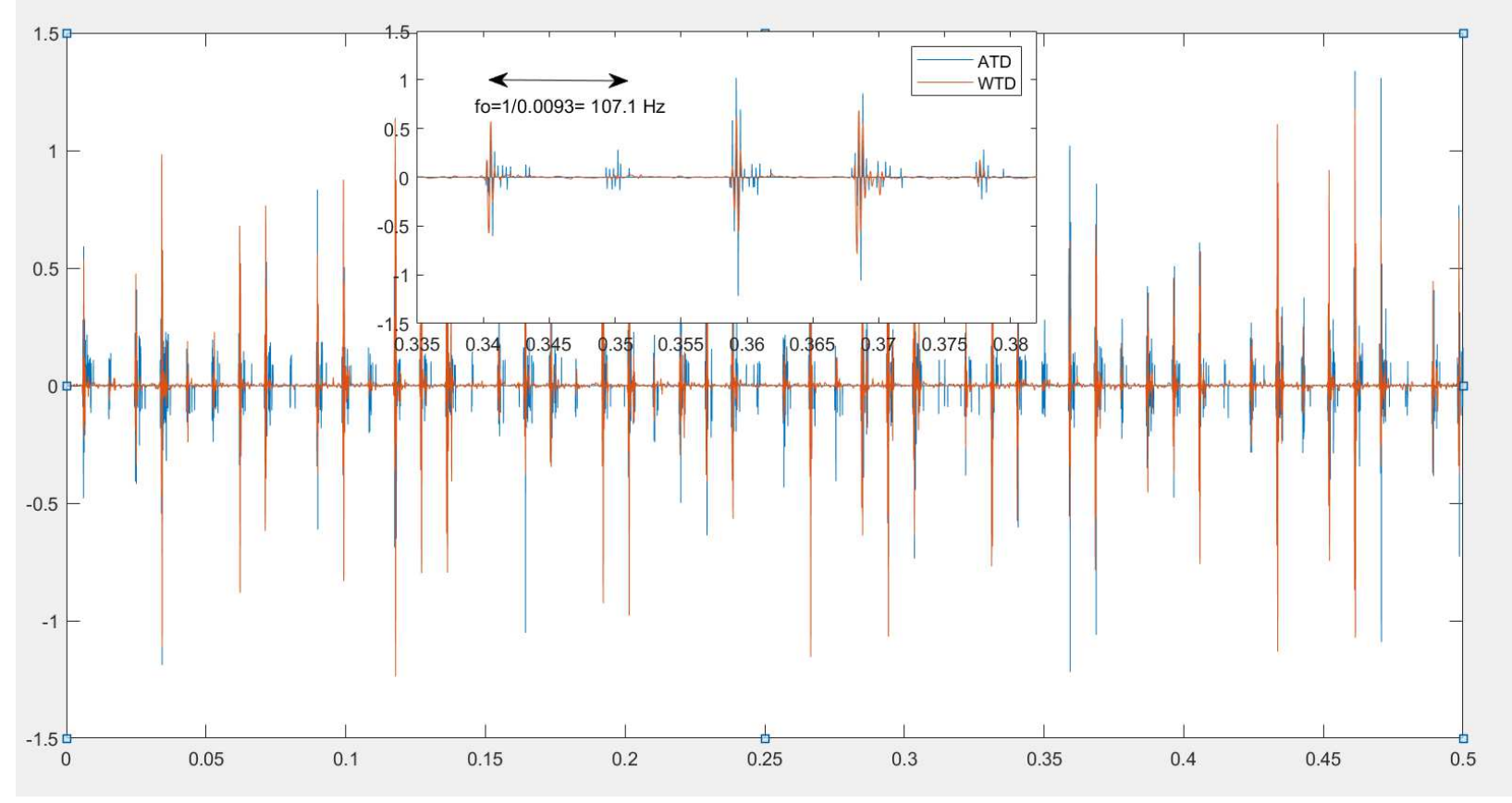

Figure 15: Outer Race fault signal denoised by WTD and ATD.

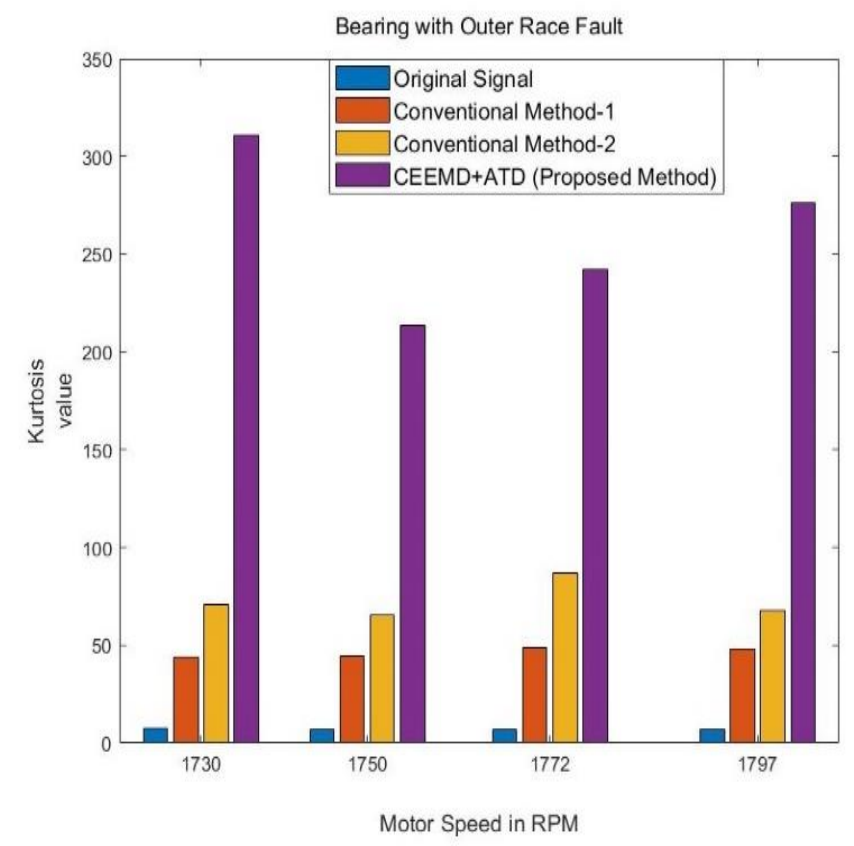

Figure 16: Kurtosis value comparison for outer race fault 

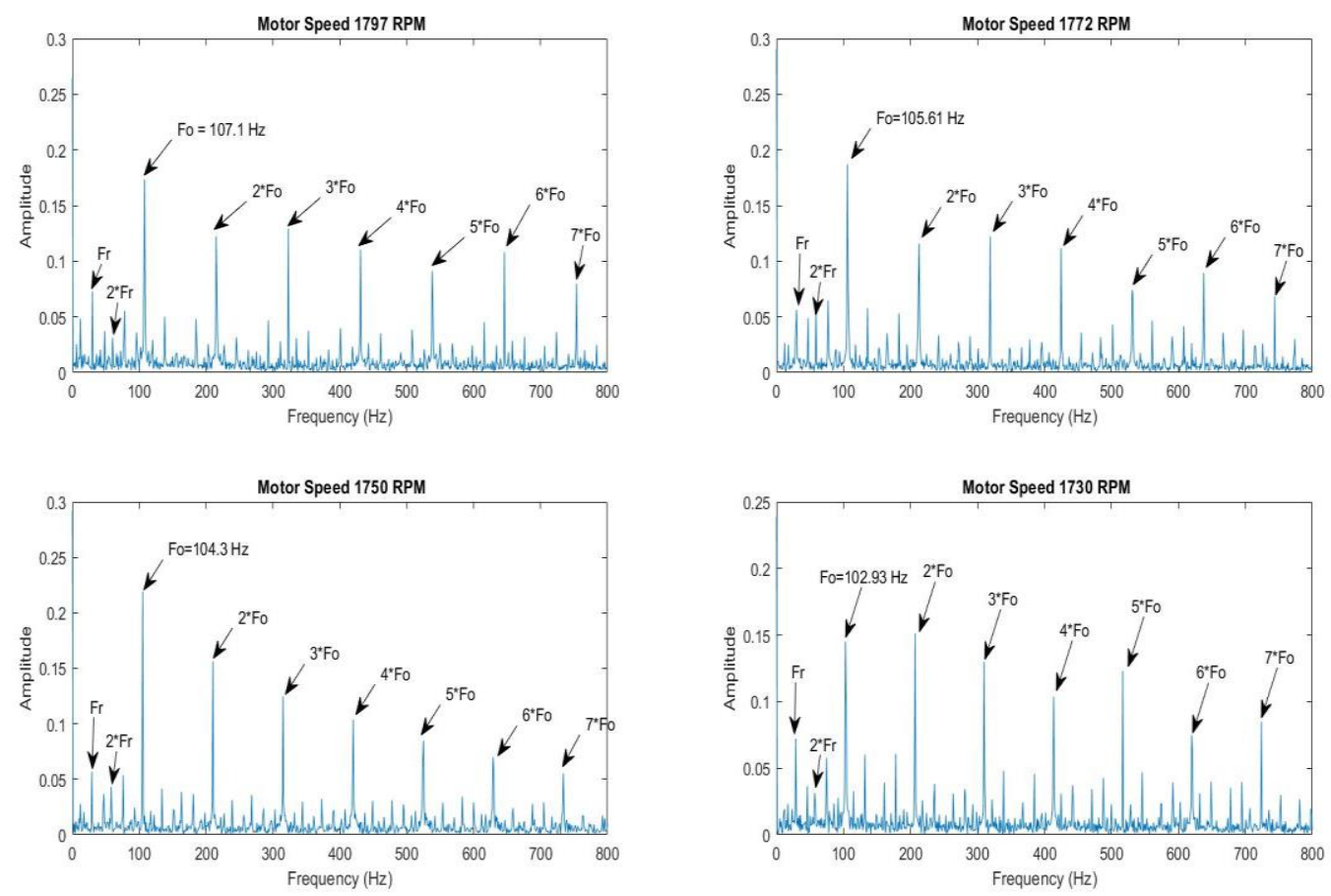

Figure 17: Envelope Spectra analysis of bearing with outer race fault signal by the proposed method.

\section{Case3: Healthy Bearing (HB) Signal}

The HB signals for motor running at a speed of 1797, 1750, 1772, and $1730 \mathrm{rpm}$, respectively, are decomposed by CEEMD and ATD. The signals denoised by proposed methods are presented in figure 18 .

The kurtosis values are calculated for denoised signals by the proposed method and compared with the two conventional methods, as shown in figure 19. The original signals kurtosis values are $2.80,2.92,2.90$, and 2.94 at rotating speeds of 1797, 1772, 1750, and $1730 \mathrm{rpm}$. The kurtosis values for the denoised signals by conventional method-1 [9] are 2.81, 2.96, 2.96, and 2.95, whereas for conventional method-2 [12] are 2.85 2.92, 2.95, and 3.05 at rotating speeds 1797, 1772, 1750, and $1730 \mathrm{rpm}$, respectively. The kurtosis value for the signals denoised by proposed methods are 3.02, 2.97, 2.98, and 3.15 at rotating speeds 1797, 1772, 1750, and $1730 \mathrm{rpm}$, respectively. The proposed method denoised signals are then subjected to an envelope spectrum 
analysis running at four different speeds, as shown in figure 20. The results obtained by the envelope spectrum analysis indicate that the peaks and harmonics appear only at the shaft rotational speed (Fr). The existence of peak at shaft rotational speed and kurtosis values close to three indicates that there is no defect in the bearing.
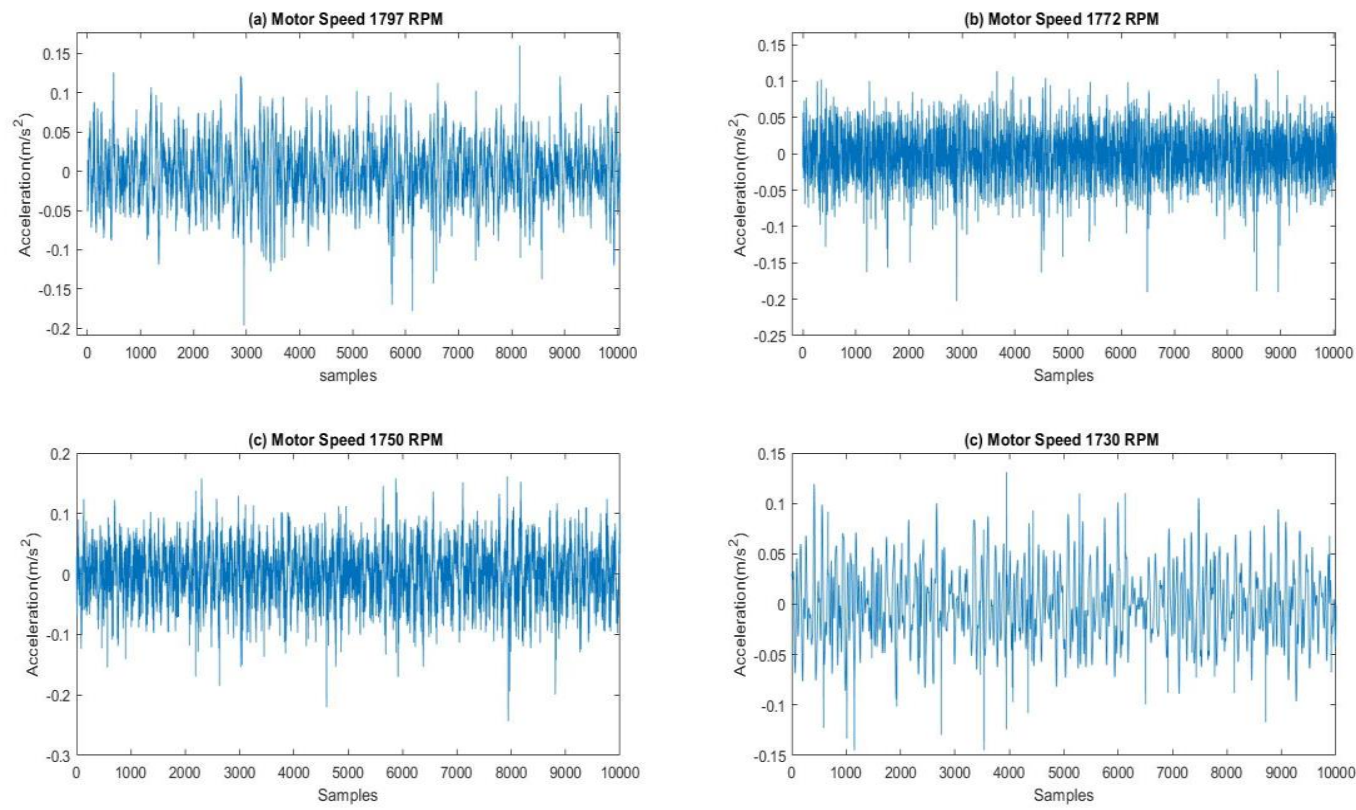

Figure 18: Denoised signals of healthy bearing running at 4 different speed by the proposed method

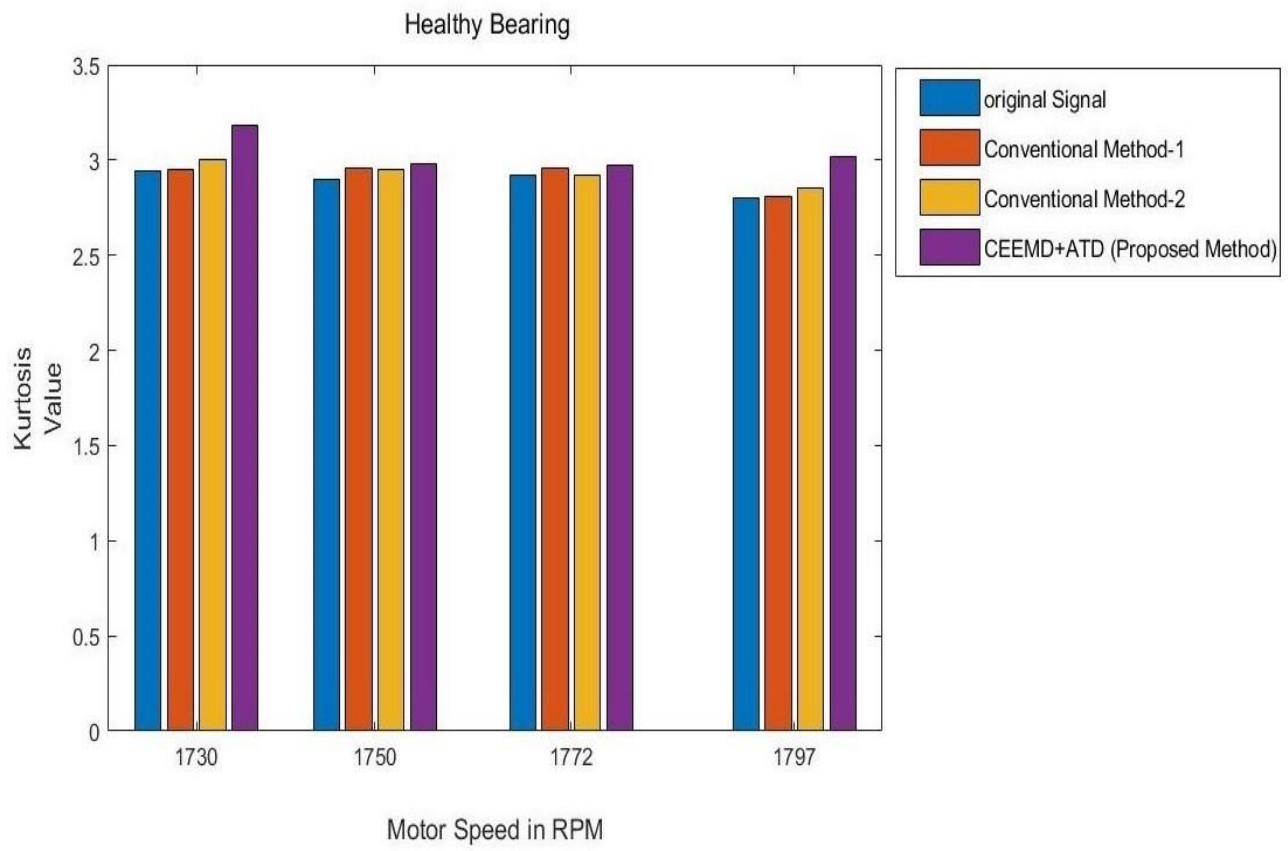

Figure 19: Kurtosis value comparison for healthy bearing 

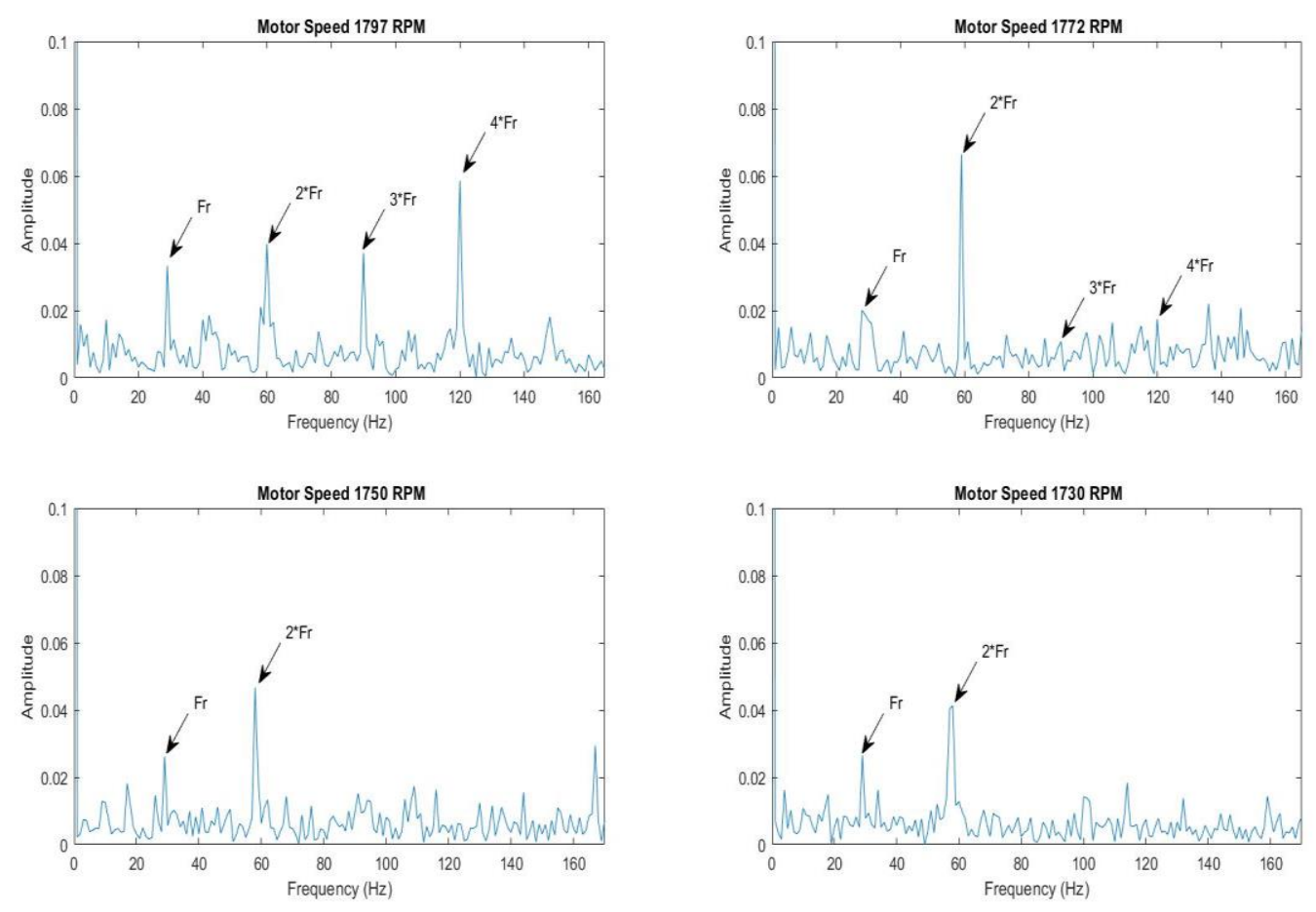

Figure 20: Envelope Spectra analysis of healthy bearing signal by the proposed method.

\section{Conclusion}

This paper presents an improved denoising method for early fault diagnosis of rolling bearing based on CEEMD and ATD methods. Firstly, the signal is decomposed by CEEMD into a various set of intrinsic mode functions (IMFs). In this method, based on correlation coefficient values, bearing IMFs are classified into four types, such as Noisy IMFs, Noise predominant IMFs, lowfrequency informative IMFs, and residuals. Noisy IMFs show an extremely high positive correlation with the original signal and are treated as noisy original signals. Therefore, it is discarded for signal reconstruction. The selected noise predominant IMFs are subjected to adaptive thresholding and added to the low-frequency IMFs to obtain a denoised signal. The kurtosis value and the envelope spectrum analysis are applied to the denoised signal to detect the presence of a fault and its location in early manner for rolling bearing. Finally, the proposed method effectiveness is measured by comparing the result with the two other conventional methods based on kurtosis value and envelope spectrum analysis. The proposed method shows significant improvement in the kurtosis parameter value compared to the two other conventional methods. 
This indicates that the proposed method is an effective technique than the other two conventional methods in identifying the defects in the rolling bearing.

\section{Declarations}

Funding: The authors received no financial support for the research, authorship, and/or publication of this article.

Conflicts of interest/Competing interests: The authors declare that they have no competing interests.

Availability of data and material: The data and material that support the findings of this study are openly available in the data repository of Case Western Reserve University.

Code availability: 'Not Applicable'

Authors' contributions: Prashant Kumar Sahu: Conceptualization, Methodology, coding, Writing - original draft, Investigation, Validation. Rajiv Nandan Rai: Data curation, Visualization, Supervision, Writing - review \& editing.

Ethics approval: 'Not Applicable’

Consent to participate: 'Not Applicable'

Consent for publication: 'Not Applicable'

\section{References}

[1] Y. Wei, Y. Li, M. Xu, and W. Huang, “A review of early fault diagnosis approaches and their applications in rotating machinery," Entropy, vol. 21, no. 4, pp. 1-26, 2019, doi: 10.3390/e21040409.

[2] M. Lebold, K. Mcclintic, R. Campbell, C. Byington, and K. Maynard, "Review of vibration 
analysis methods for gearbox diagnostics and prognostics," 54th Meet. Soc. Mach. Fail. Prev. Technol., pp. 623-634, 2000.

[3] R. B. W. Heng and M. J. M. Nor, "Statistical analysis of sound and vibration signals for monitoring rolling element bearing condition," Appl. Acoust., vol. 53, no. 1-3, pp. 211-226, 1998, doi: 10.1016/s0003-682x(97)00018-2.

[4] H. R. Martin and F. Honarvar, "Application of statistical moments to bearing failure detection," Appl. Acoust., vol. 44, no. 1, pp. 67-77, 1995, doi: 10.1016/0003682X(94)P4420-B.

[5] J. Antoni and R. B. Randall, "The spectral kurtosis: Application to the vibratory surveillance and diagnostics of rotating machines," Mech. Syst. Signal Process., vol. 20, no. 2, pp. 308 331, 2006, doi: 10.1016/j.ymssp.2004.09.002.

[6] P. D. McFadden and J. D. Smith, "Vibration monitoring of rolling element bearings by the high-frequency resonance technique - a review," Tribol. Int., vol. 17, no. 1, pp. 3-10, 1984, doi: 10.1016/0301-679X(84)90076-8.

[7] N. E. Huang et al., "The empirical mode decomposition and the Hubert spectrum for nonlinear and non-stationary time series analysis," Proc. R. Soc. A Math. Phys. Eng. Sci., vol. 454, no. 1971, pp. 903-995, 1998, doi: 10.1098/rspa.1998.0193.

[8] V. K. Rai and A. R. Mohanty, "Bearing fault diagnosis using FFT of intrinsic mode functions in Hilbert-Huang transform," Mech. Syst. Signal Process., vol. 21, no. 6, pp. 2607-2615, 2007, doi: 10.1016/j.ymssp.2006.12.004.

[9] D. zun Zhao, J. yong Li, W. dong Cheng, T. yang Wang, and W. gang Wen, "Rolling element bearing instantaneous rotational frequency estimation based on EMD softthresholding denoising and instantaneous fault characteristic frequency," J. Cent. South Univ., vol. 23, no. 7, pp. 1682-1689, 2016, doi: 10.1007/s11771-016-3222-x.

[10] A. Ayenu-prah and N. I. I. Attoh-okine, "Intrinsic Mode Functions in Empirical Mode Decomposition," Analysis, vol. 2, no. 1, pp. 1-24, 2010.

[11] K. Zhu, X. Song, and D. Xue, "Incipient fault diagnosis of roller bearings using empirical mode decomposition and correlation coefficient.," J. Vibroengineering, vol. 15, no. 2, pp. 597-603, Jun. [Online]. 2013, Available: http://search.ebscohost.com/login.aspx?direct=true $\& \mathrm{db}=\mathrm{asx} \& \mathrm{AN}=88875824 \&$ site=edslive. 
[12] R. Abdelkader, A. Kaddour, and Z. Derouiche, "Enhancement of rolling bearing fault diagnosis based on improvement of empirical mode decomposition denoising method," Int. J. Adv. Manuf. Technol., vol. 97, no. 5-8, pp. 3099-3117, 2018, doi: 10.1007/s00170-0182167-7.

[13] R. Abdelkader, A. Kaddour, A. Bendiabdellah, and Z. Derouiche, "Rolling Bearing Fault Diagnosis Based on an Improved Denoising Method Using the Complete Ensemble Empirical Mode Decomposition and the Optimized Thresholding Operation," IEEE Sens. J., vol. 18, no. 17, pp. 7166-7172, 2018, doi: 10.1109/JSEN.2018.2853136.

[14] G. Rilling and P. Flandrin, "On the influence of sampling on the empirical mode decomposition," in 2006 IEEE International Conference on Acoustics Speech and Signal Processing Proceedings, 2006, vol. 3, pp. III-III.

[15] Z. Wu and N. E. Huang, "Ensemble empirical mode decomposition: a noise-assisted data analysis method," Adv. Adapt. Data Anal., vol. 1, no. 01, pp. 1-41, 2009.

[16] Y. Lei, Z. He, and Y. Zi, "Application of the EEMD method to rotor fault diagnosis of rotating machinery," Mech. Syst. Signal Process., vol. 23, no. 4, pp. 1327-1338, 2009, doi: 10.1016/j.ymssp.2008.11.005.

[17] M. Li, H. Wang, G. Tang, H. Yuan, and Y. Yang, "An improved method based on CEEMD for fault diagnosis of rolling bearing," Adv. Mech. Eng., vol. 2014, no. November 2014, 2014, doi: 10.1155/2014/676205.

[18] Y. Lu, R. Xie, and S. Y. Liang, "CEEMD-assisted kernel support vector machines for bearing diagnosis," Int. J. Adv. Manuf. Technol., vol. 106, no. 7-8, pp. 3063-3070, 2020, doi: 10.1007/s00170-019-04858-w.

[19] F. Liu, J. Gao, and H. Liu, "The Feature Extraction and Diagnosis of Rolling Bearing Based on CEEMD and LDWPSO-PNN," vol. 8, 2020.

[20] Z. Wei, Y. Wang, S. He, and J. Bao, "A novel intelligent method for bearing fault diagnosis based on affinity propagation clustering and adaptive feature selection," Knowledge-Based Syst., vol. 116, pp. 1-12, 2017.

[21] S. N. Chegini, A. Bagheri, and F. Najafi, "Application of a new EWT-based denoising technique in bearing fault diagnosis," Meas. J. Int. Meas. Confed., vol. 144, pp. 275-297, 2019, doi: 10.1016/j.measurement.2019.05.049.

[22] M. Yabin, C. Chen, S. Qiqi, W. Jian, L. Hongliang, and H. Darong, "Fault diagnosis of 
rolling bearing based on EMD combined with HHT envelope and wavelet spectrum transform," Proc. 2018 IEEE 7th Data Driven Control Learn. Syst. Conf. DDCLS 2018, pp. 481-485, 2018, doi: 10.1109/DDCLS.2018.8516038.

[23] M. Zhang and G. Wei, "An integrated EMD adaptive threshold denoising method for reduction of noise in ECG," PLoS One, vol. 15, no. 7 July, pp. 1-30, 2020, doi: 10.1371/journal.pone.0235330.

[24] "Bearing Data Center - Case Western Reserve University," https://csegroups.case.edu/bearingdatacenter/pages/welcome-case-western-reserveuniversity-bearing-data-center-website. . 
Figures

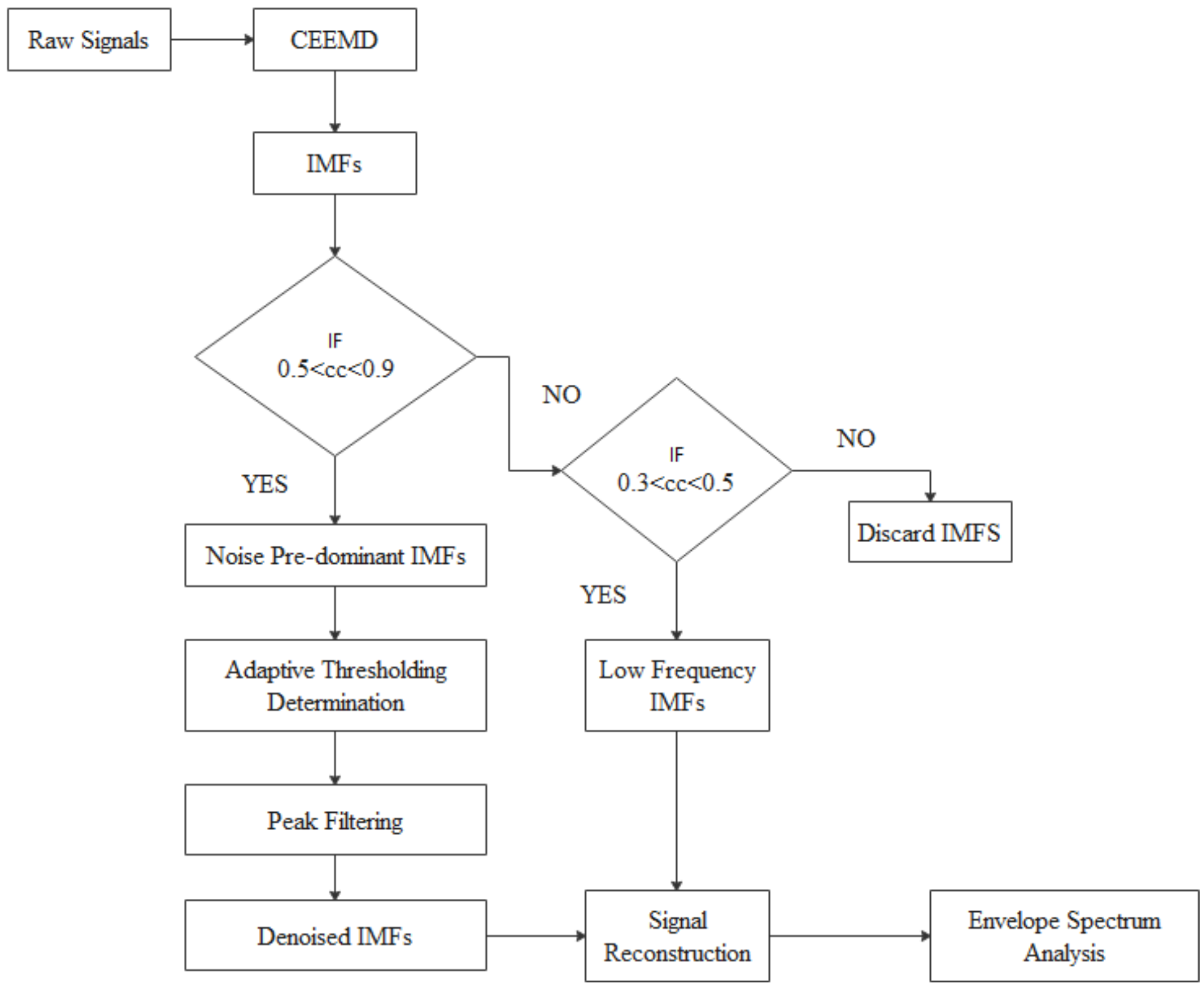

\section{Figure 1}

Flow Chart of CEEMD-ATD (Proposed method) for Bearing Fault Diagnosis. 


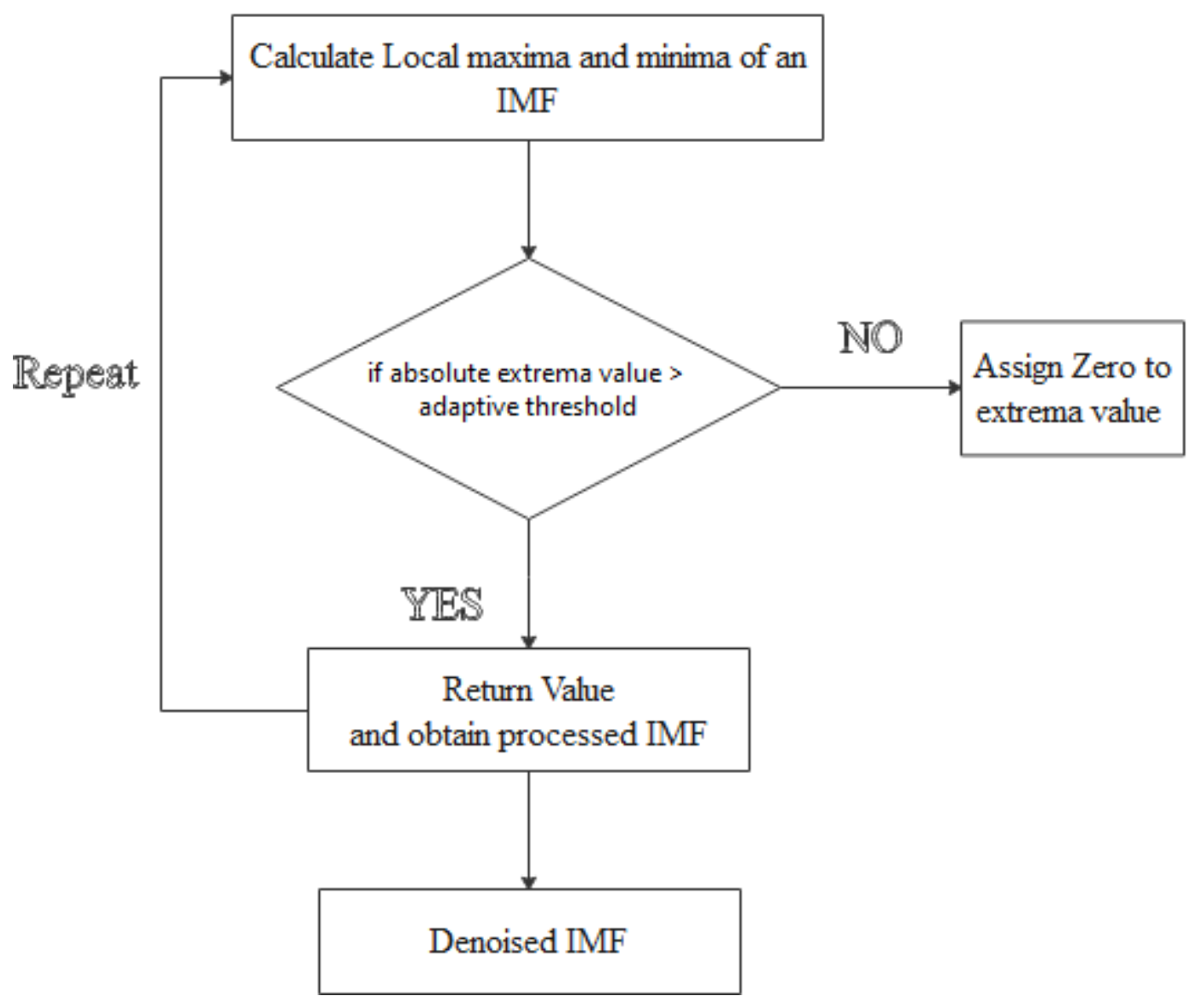

Figure 2

Flow chart shows the working of the peak filtering denoising Method.

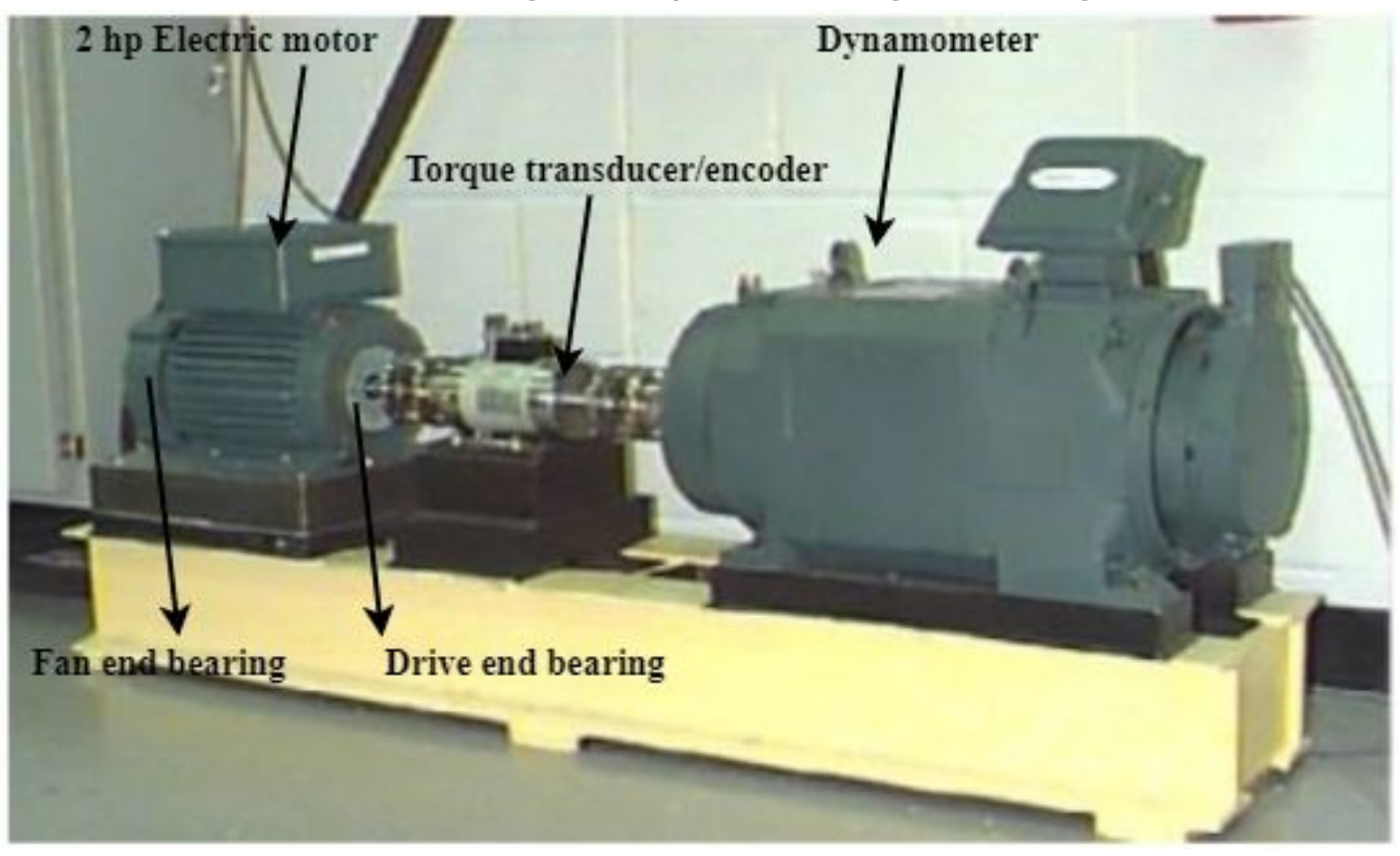

Figure 3

CWRU bearing test rig [24] 
(a) Healthy Bearing

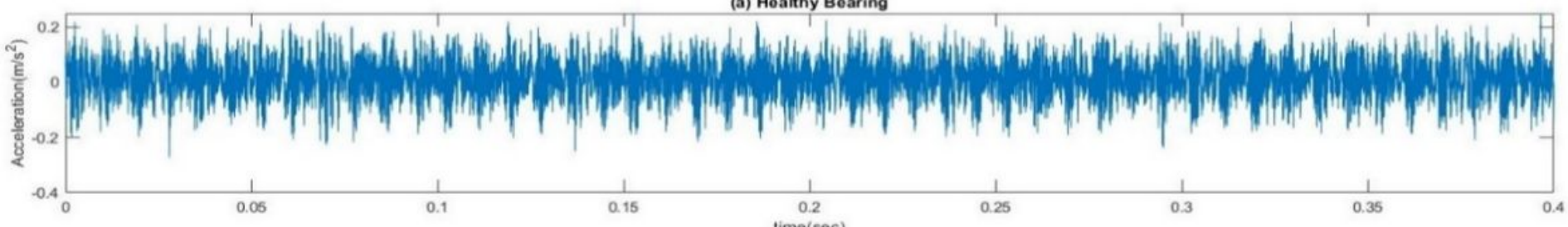

(b) Bearing with Inner Race Fault

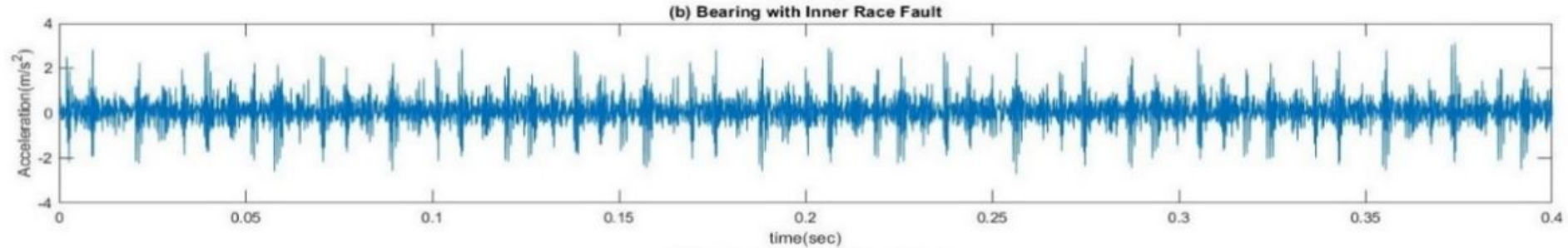

(c) Bearing with Outer Race Fault

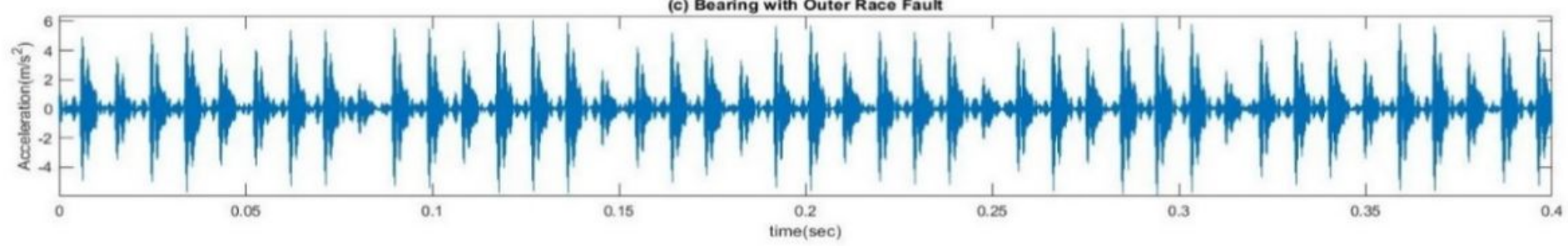

\section{Figure 4}

Original Signals (a) Healthy Bearing (b) Bearing with Inner Race Fault and (c) Bearing with Outer Race Fault
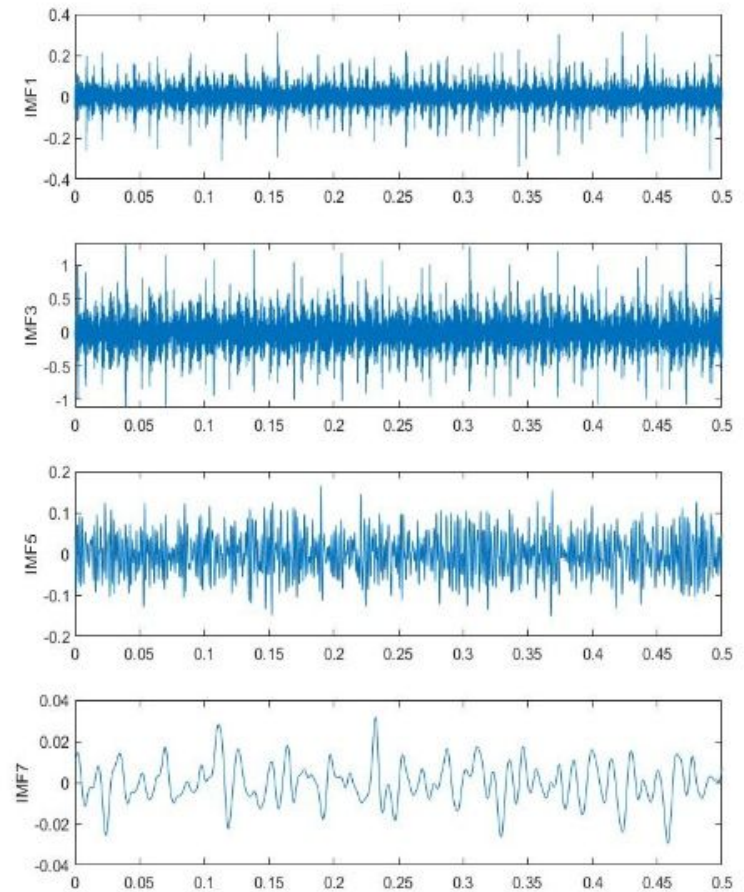
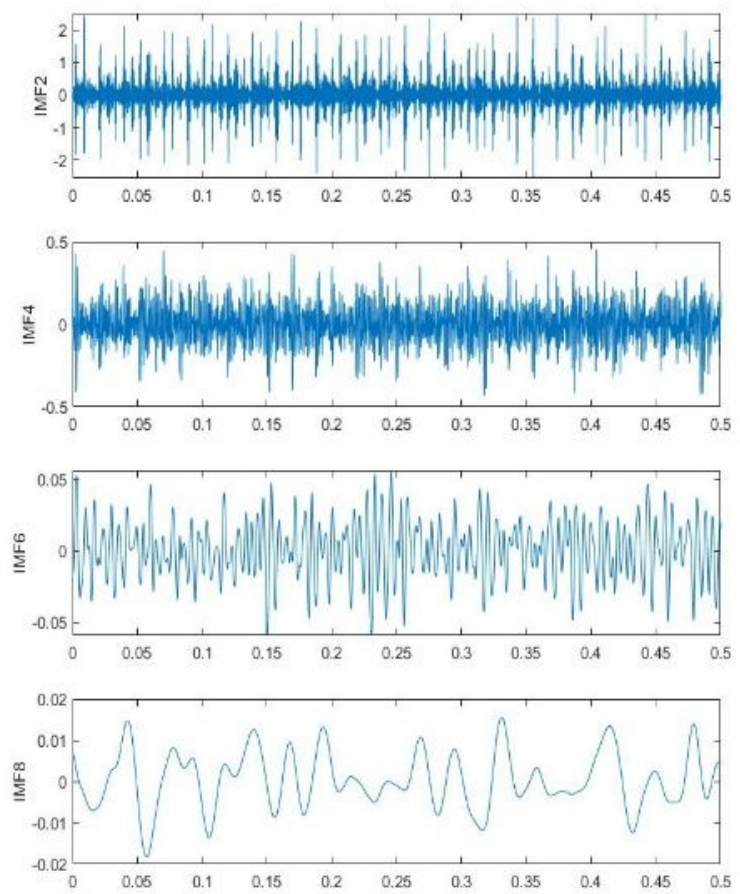

\section{Figure 5}




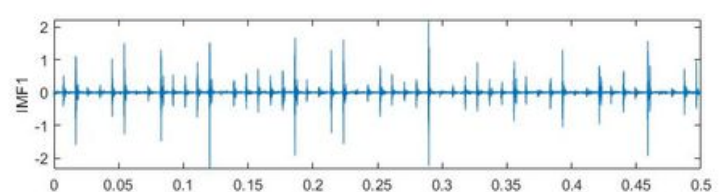

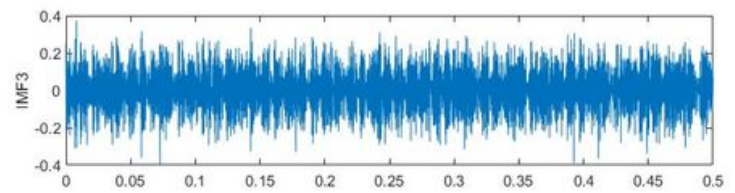
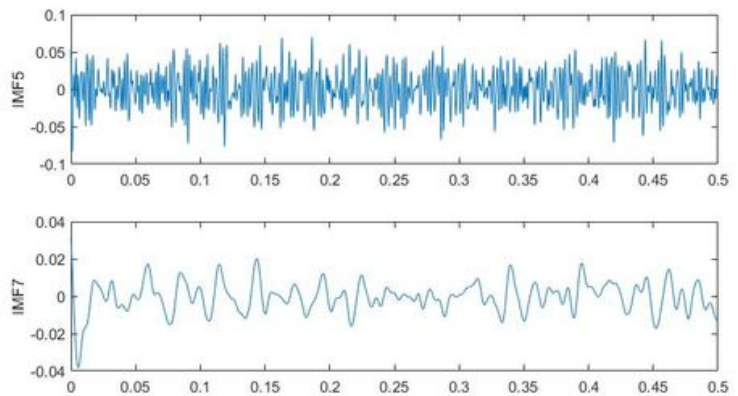

\section{Figure 6}

Outer race fault signal decomposed by CEEMD
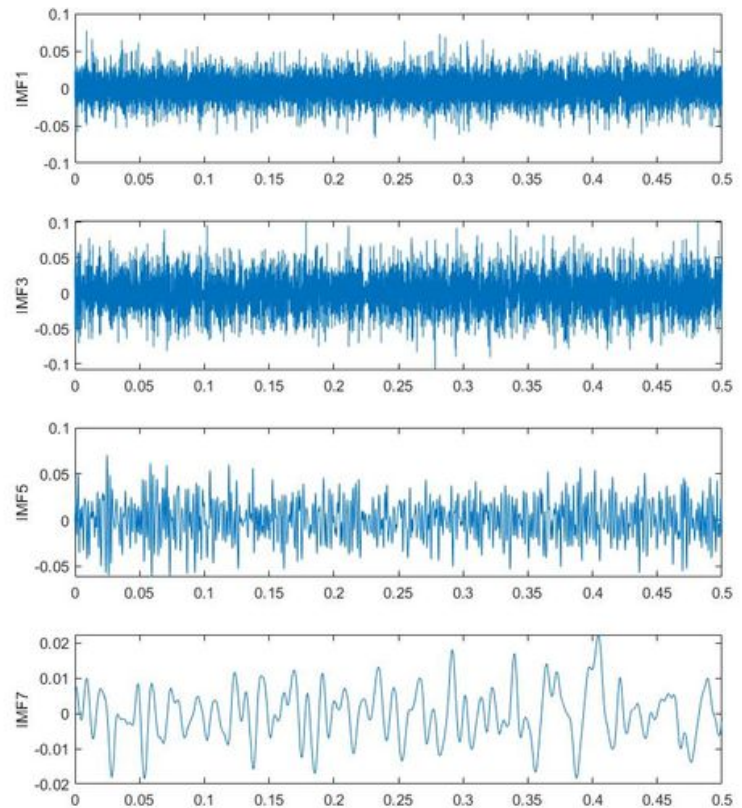
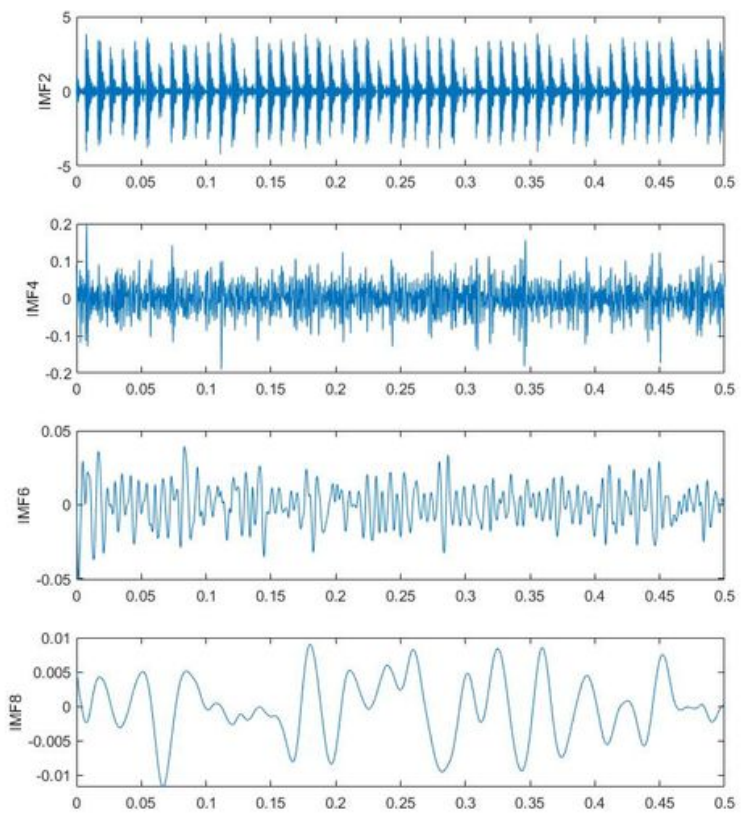

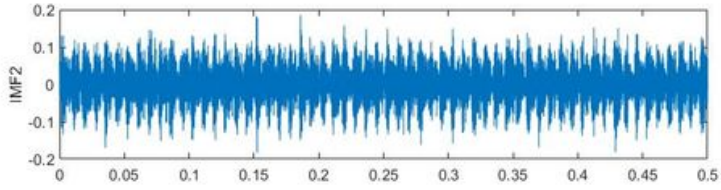
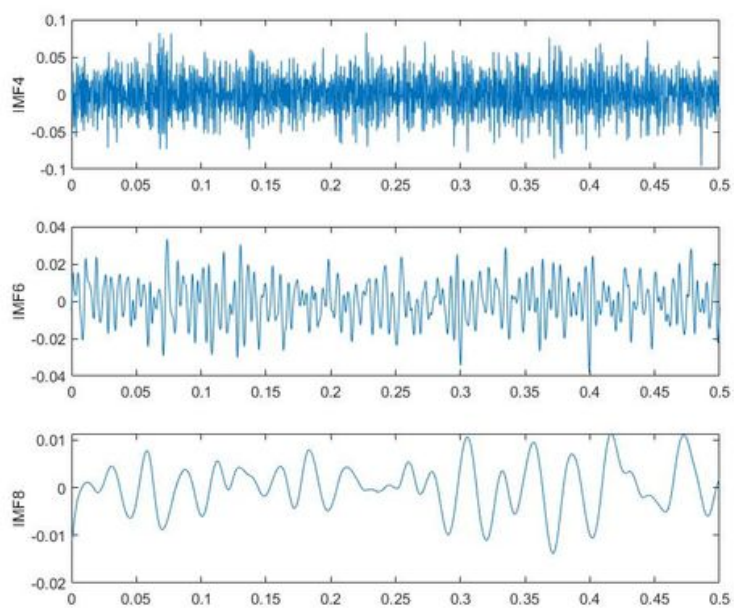

Figure 7

Healthy bearing signal decomposed by CEEMD 


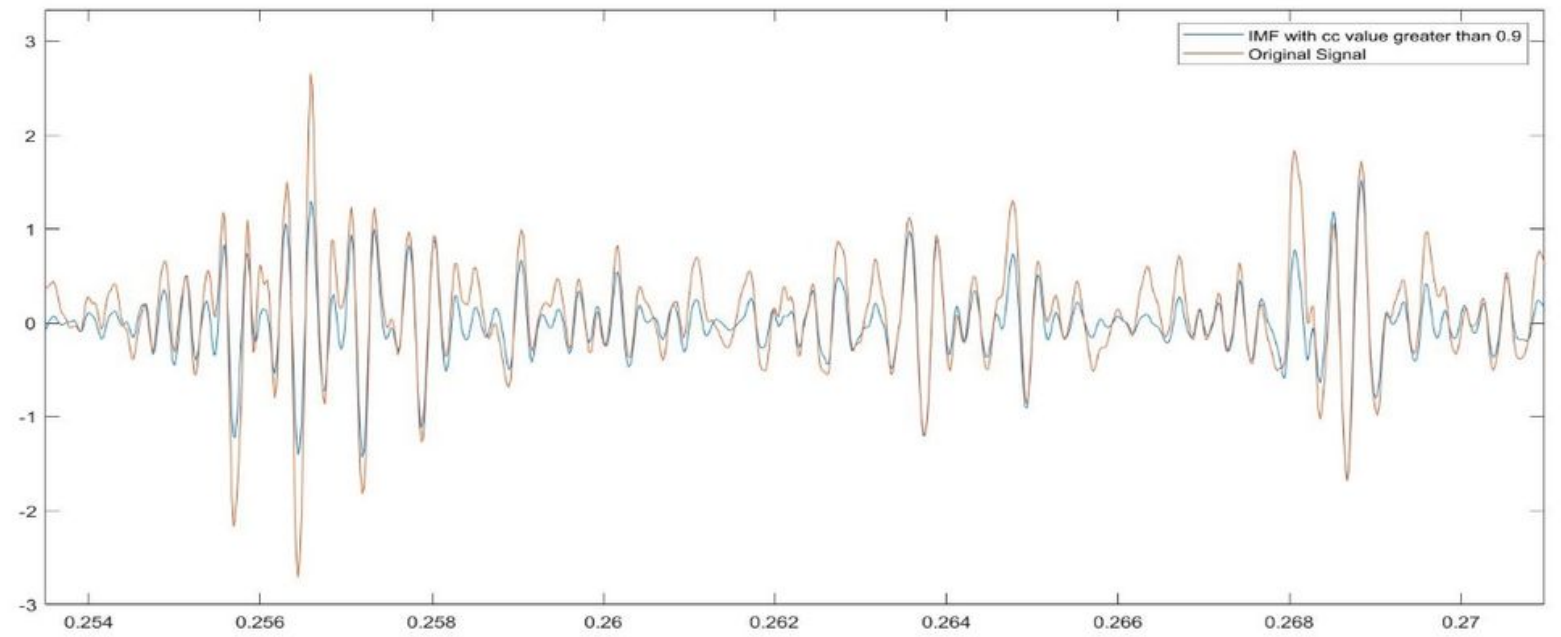

Figure 8

IMF vs Original signal for Inner race fault

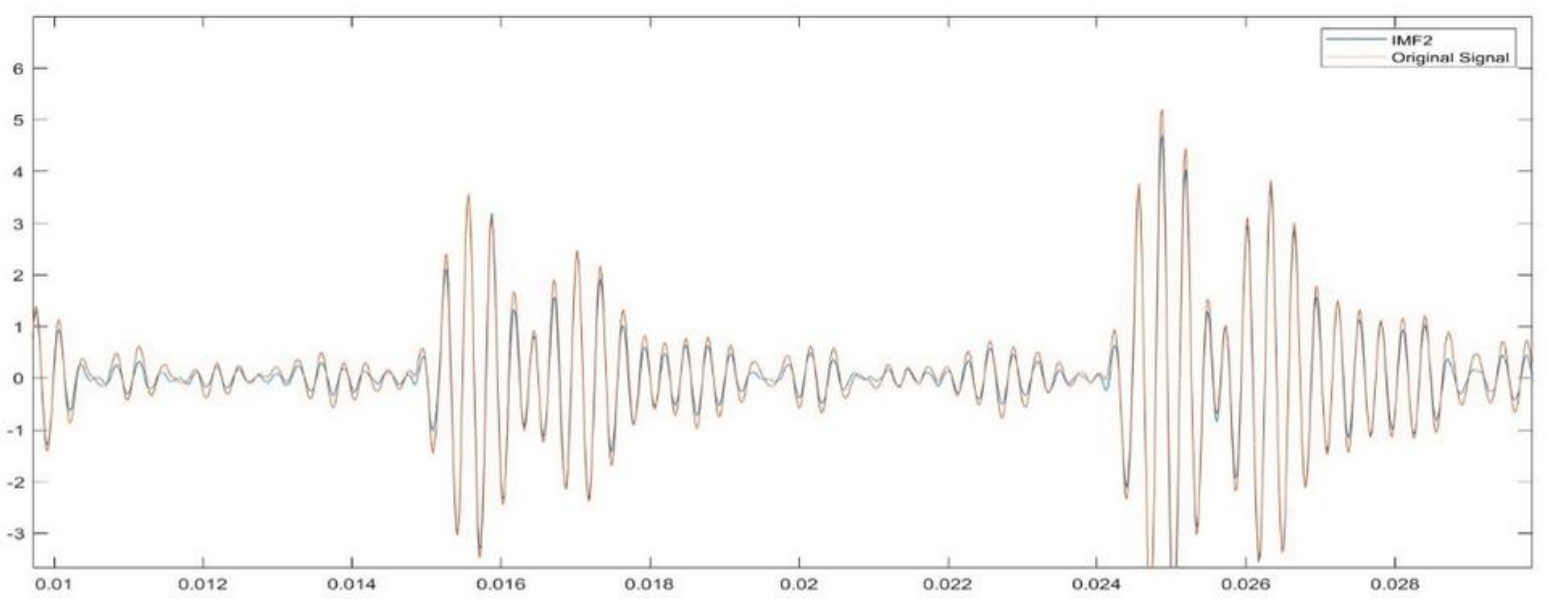

Figure 9

IMF2 vs Original signal for outer race fault 

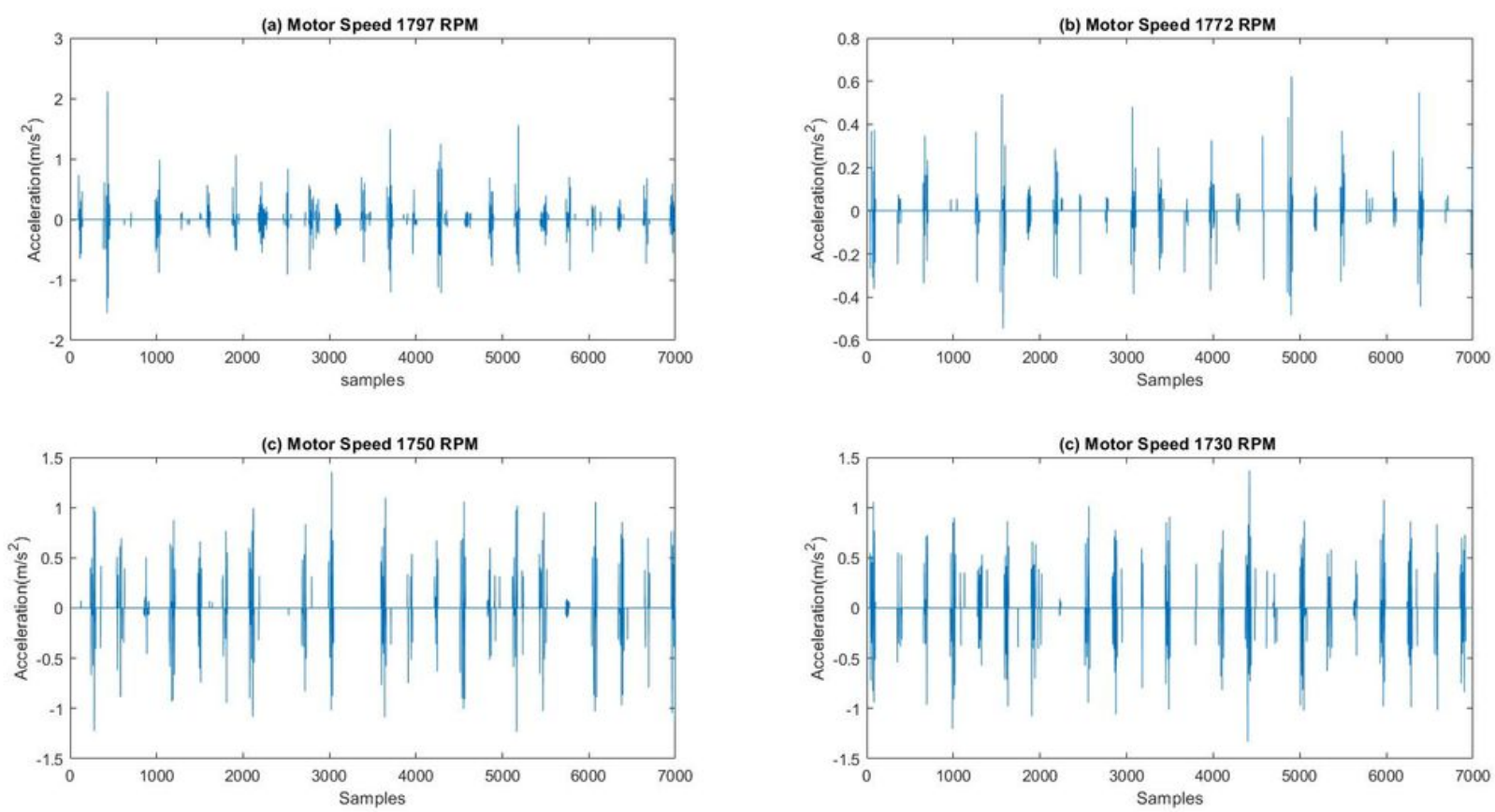

Figure 10

Denoised signals of bearing with inner race fault running at 4 different speed by the proposed method

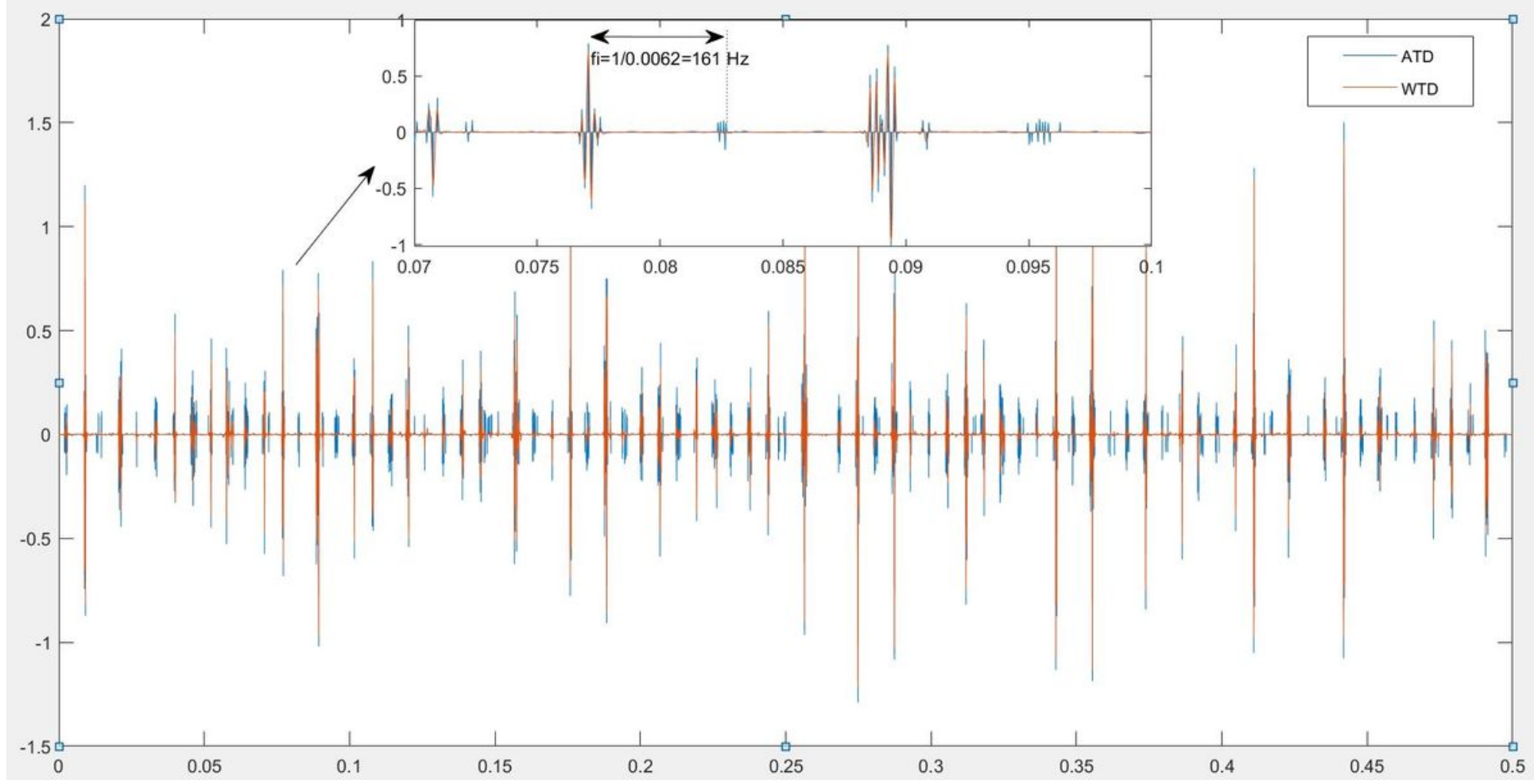

Figure 11

Inner Race fault signal denoised by WTD and ATD. 


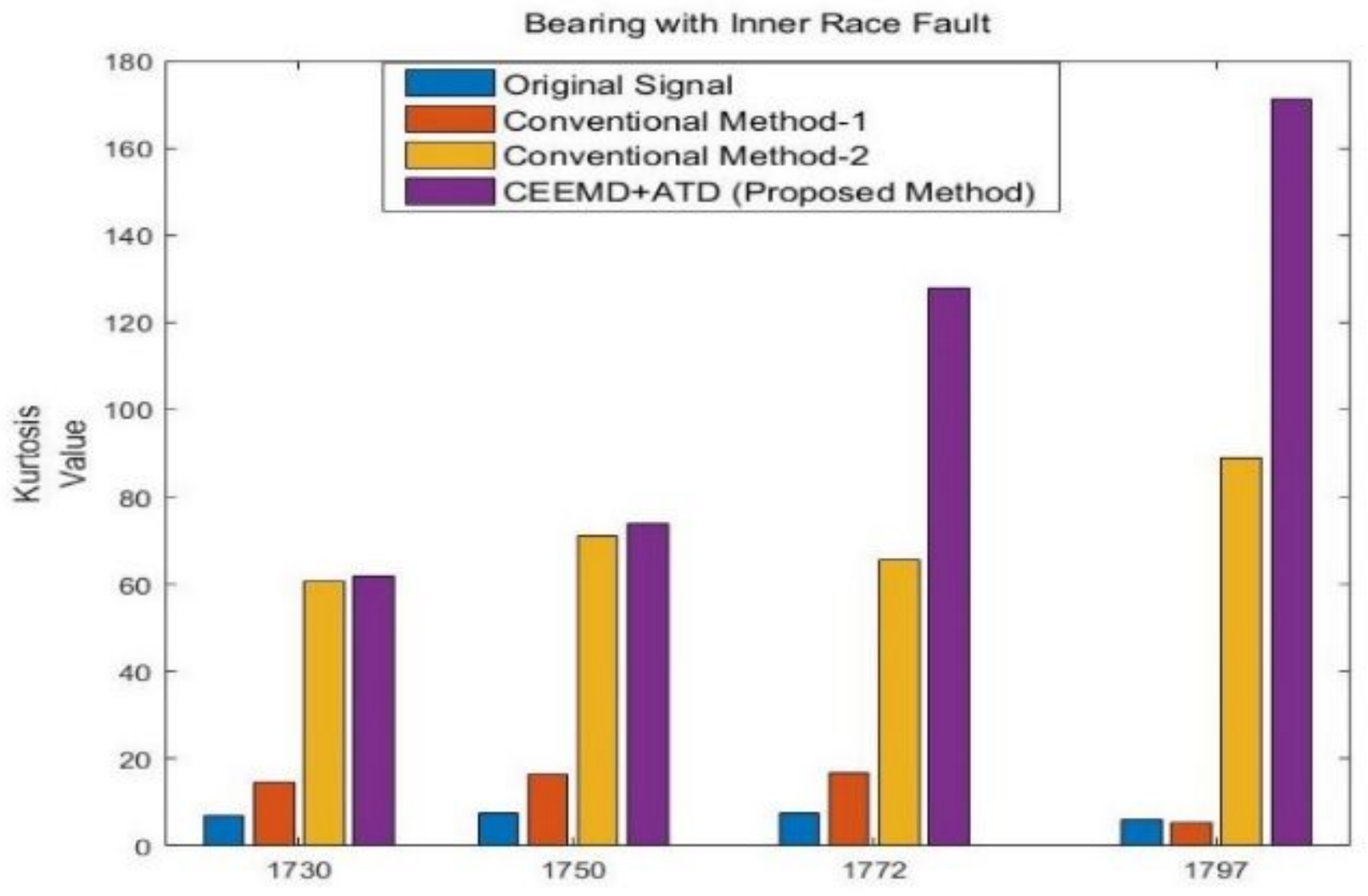

Motor Speed in RPM

Figure 12

Kurtosis value comparison for Inner race fault
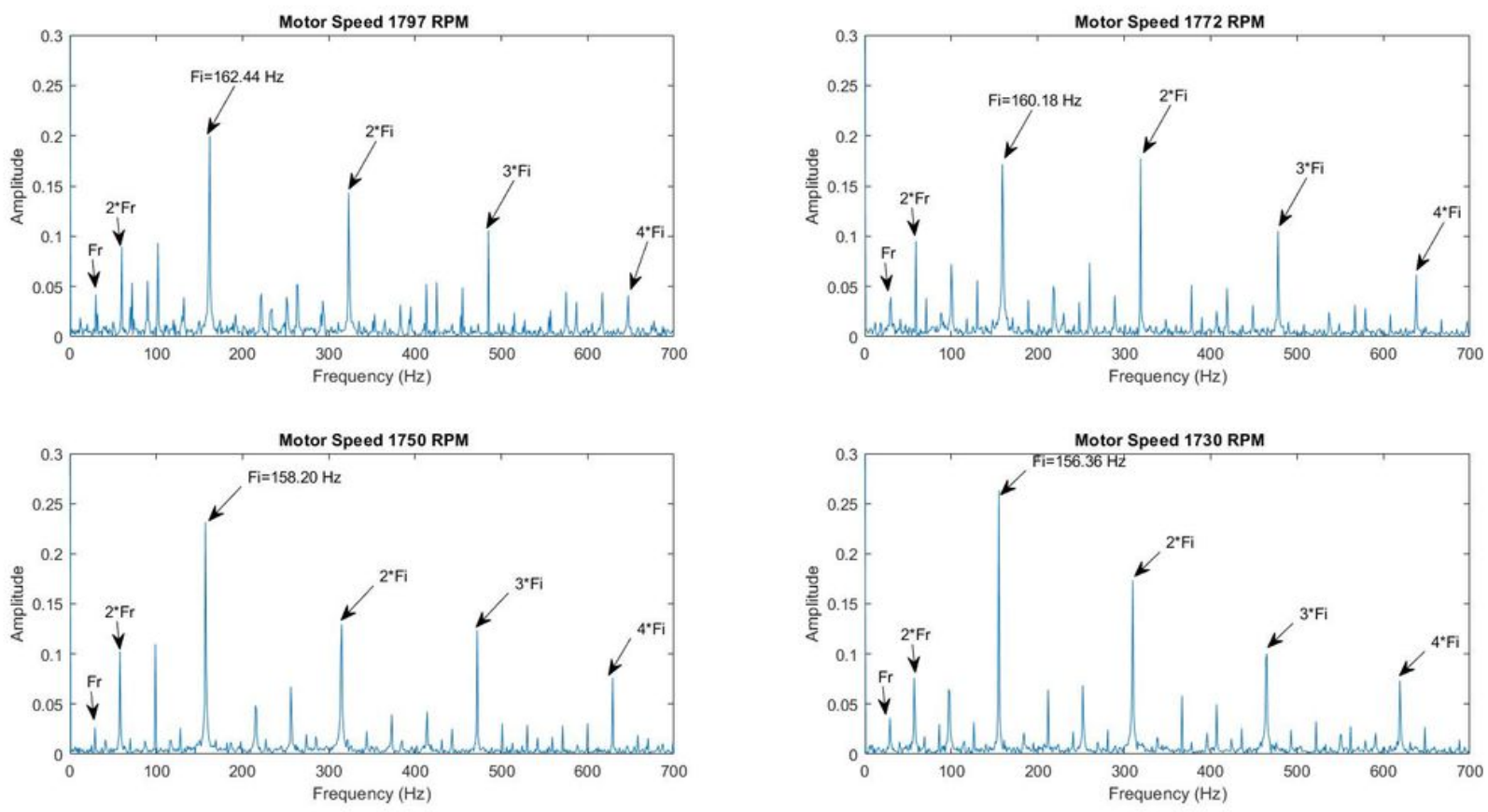

Figure 13 
Envelope Spectra analysis of bearing with inner race fault signal by the proposed method.
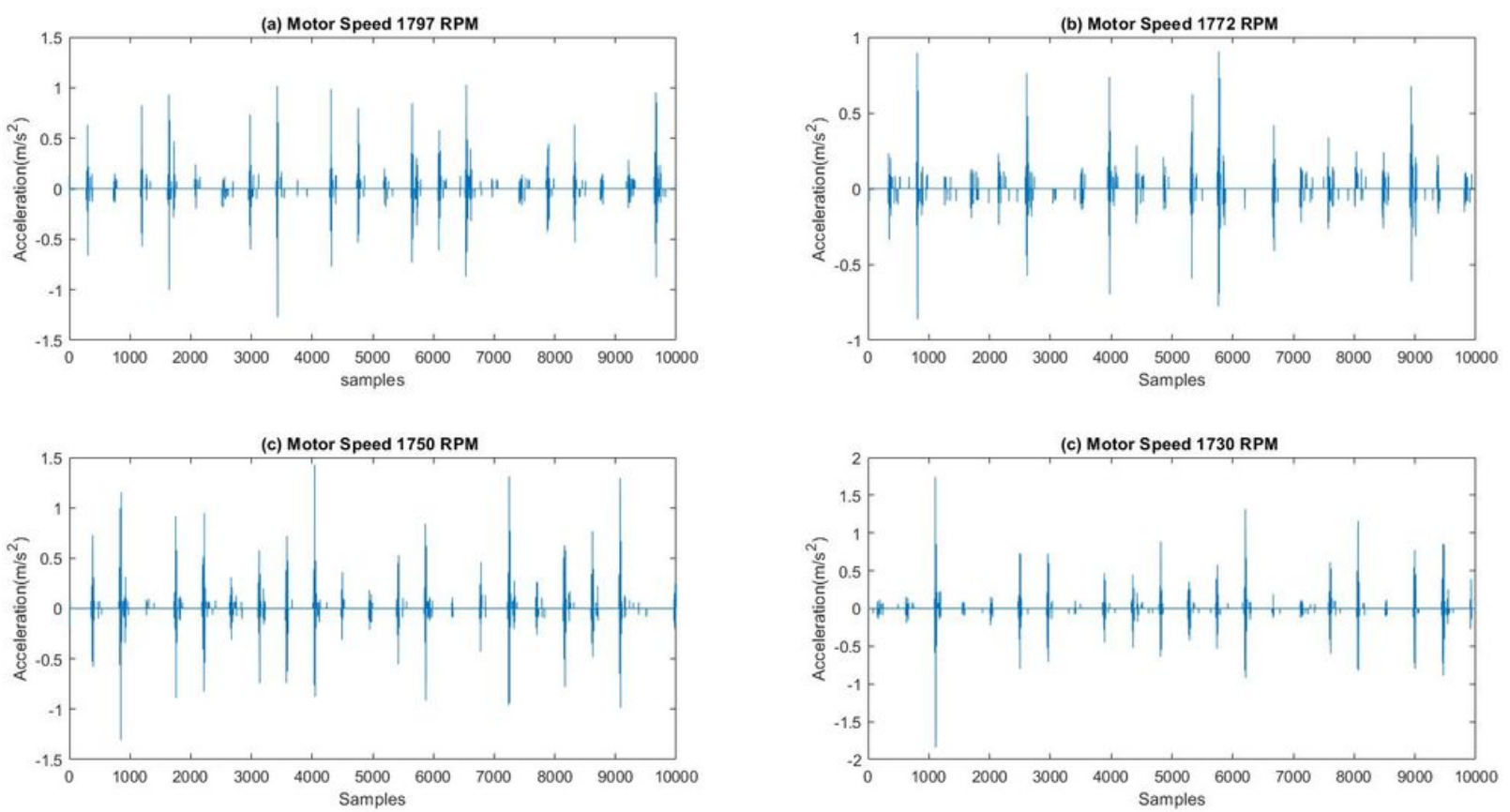

\section{Figure 14}

Denoised signals of bearing with outer race fault running at 4 different speed by the proposed method

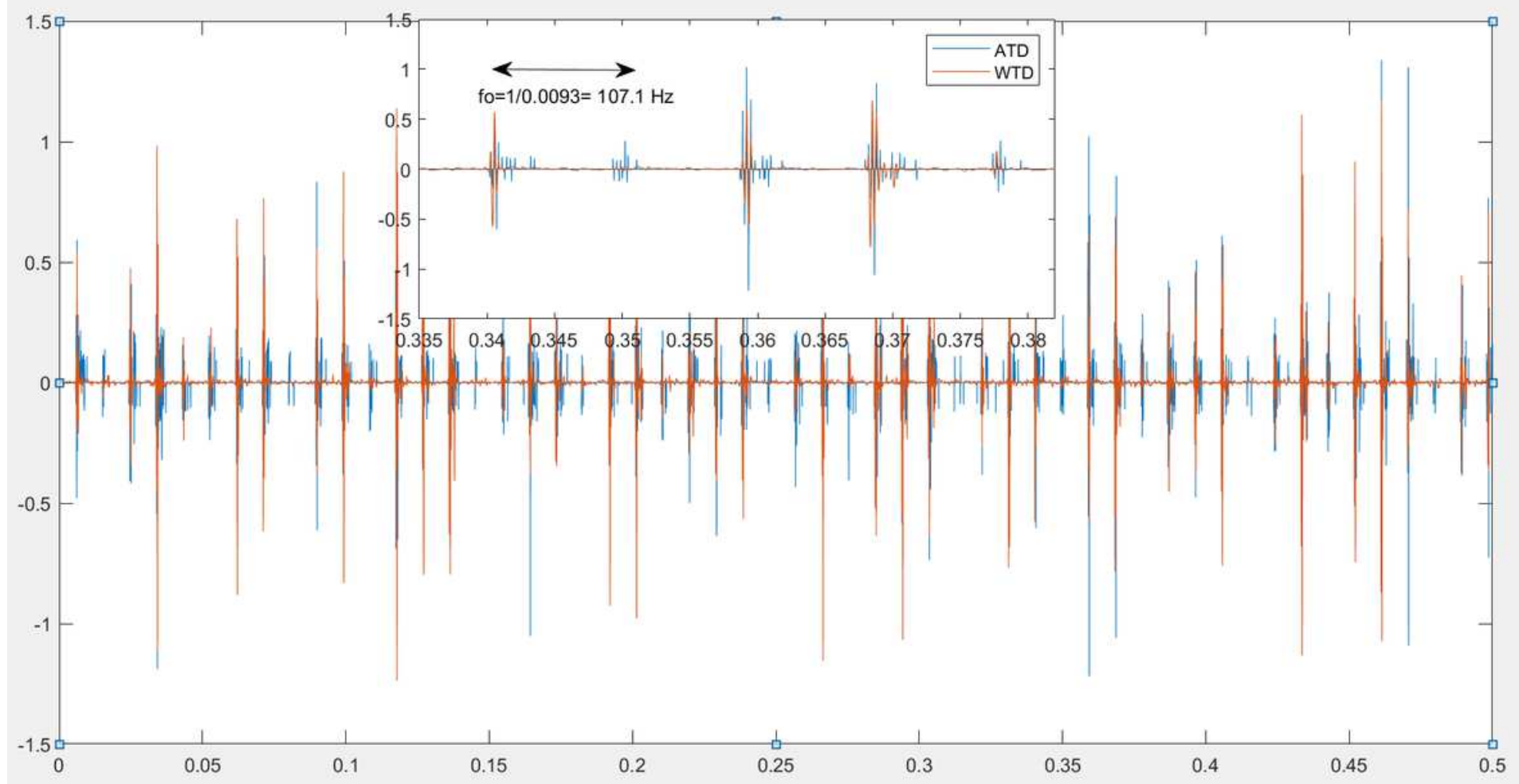

Figure 15

Outer Race fault signal denoised by WTD and ATD. 
Bearing with Outer Race Fault

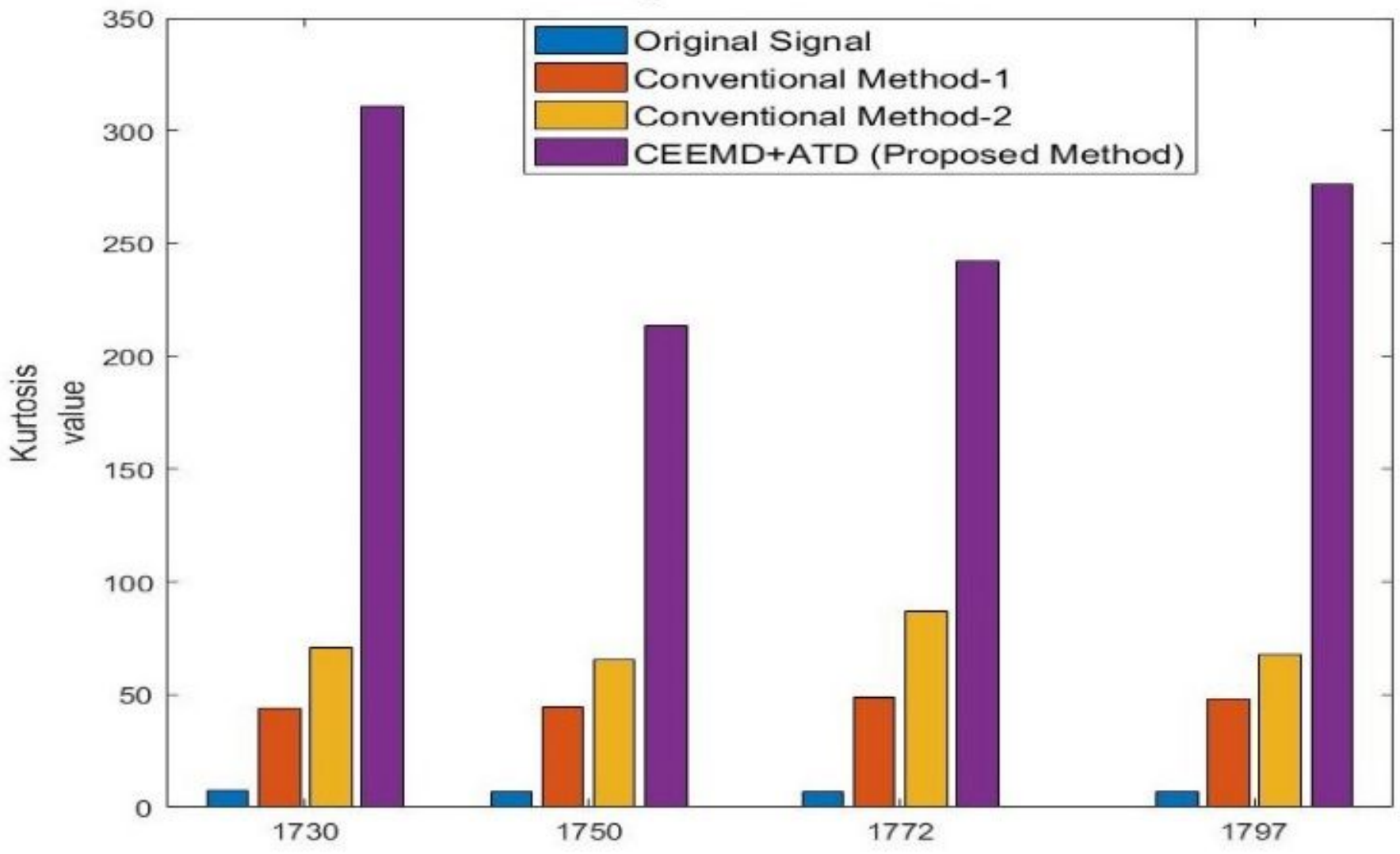

Motor Speed in RPM

Figure 16

Kurtosis value comparison for outer race fault
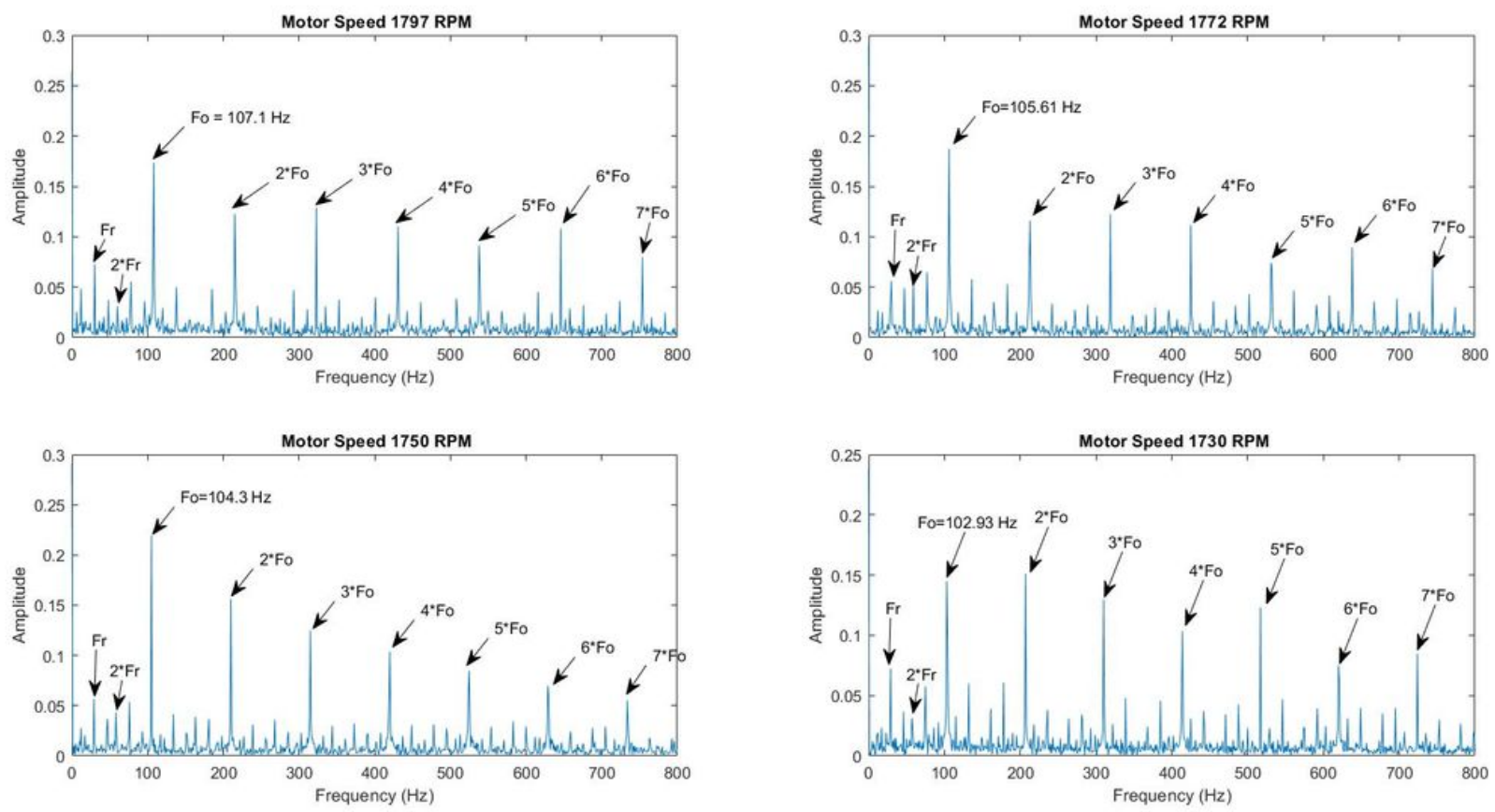
Figure 17

Envelope Spectra analysis of bearing with outer race fault signal by the proposed method.
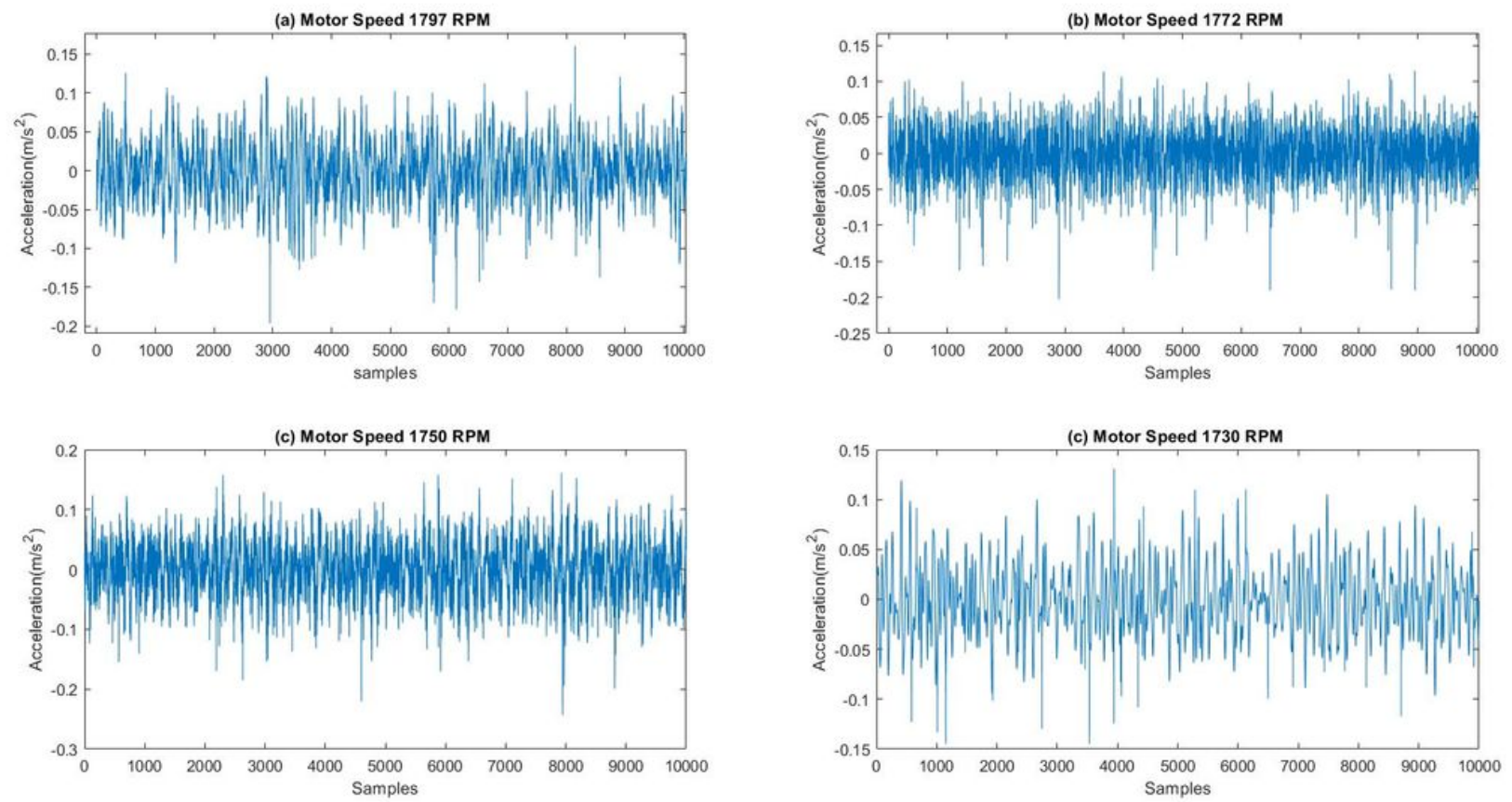

Figure 18

Denoised signals of healthy bearing running at 4 different speed by the proposed method Healthy Bearing

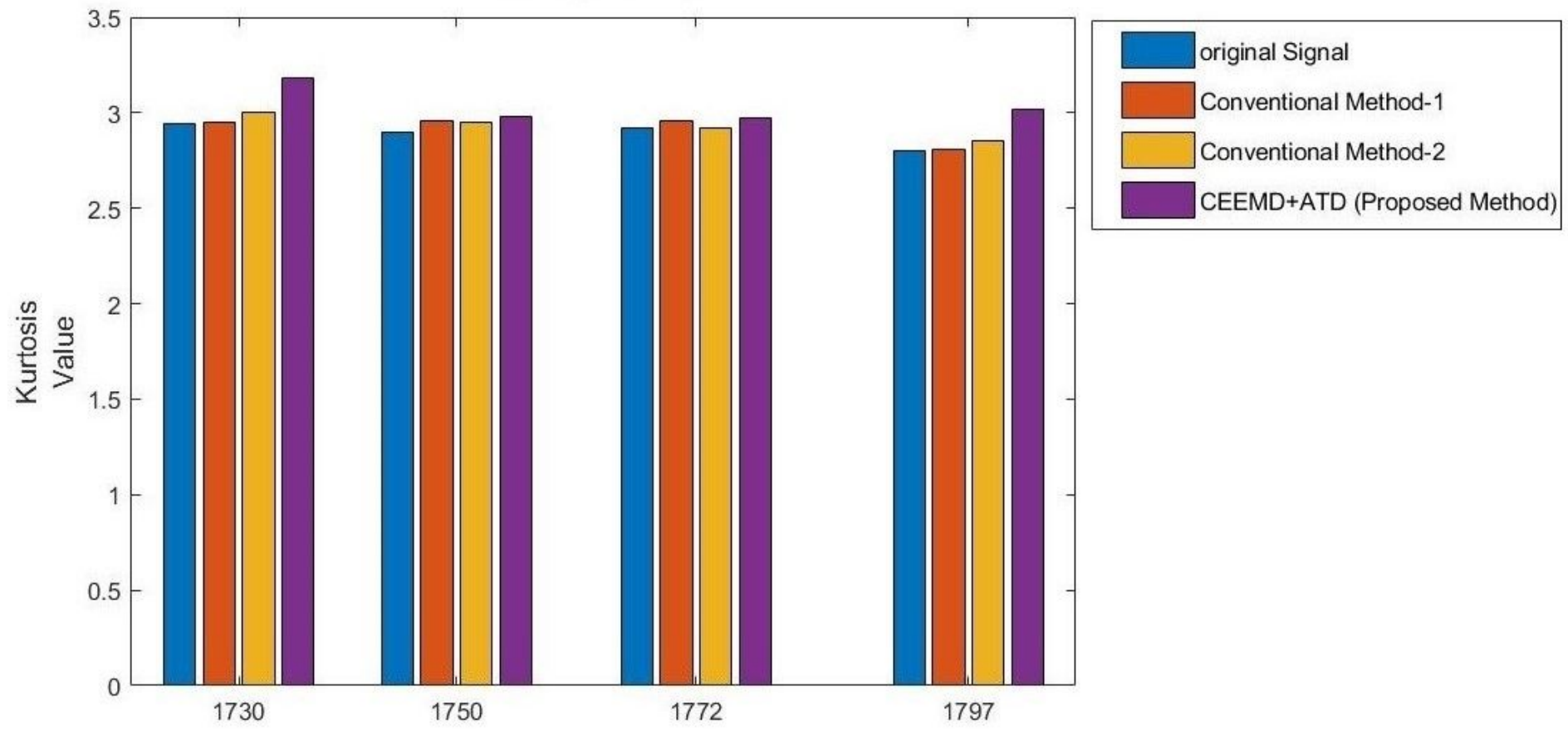

Motor Speed in RPM

Figure 19 
Kurtosis value comparison for healthy bearing
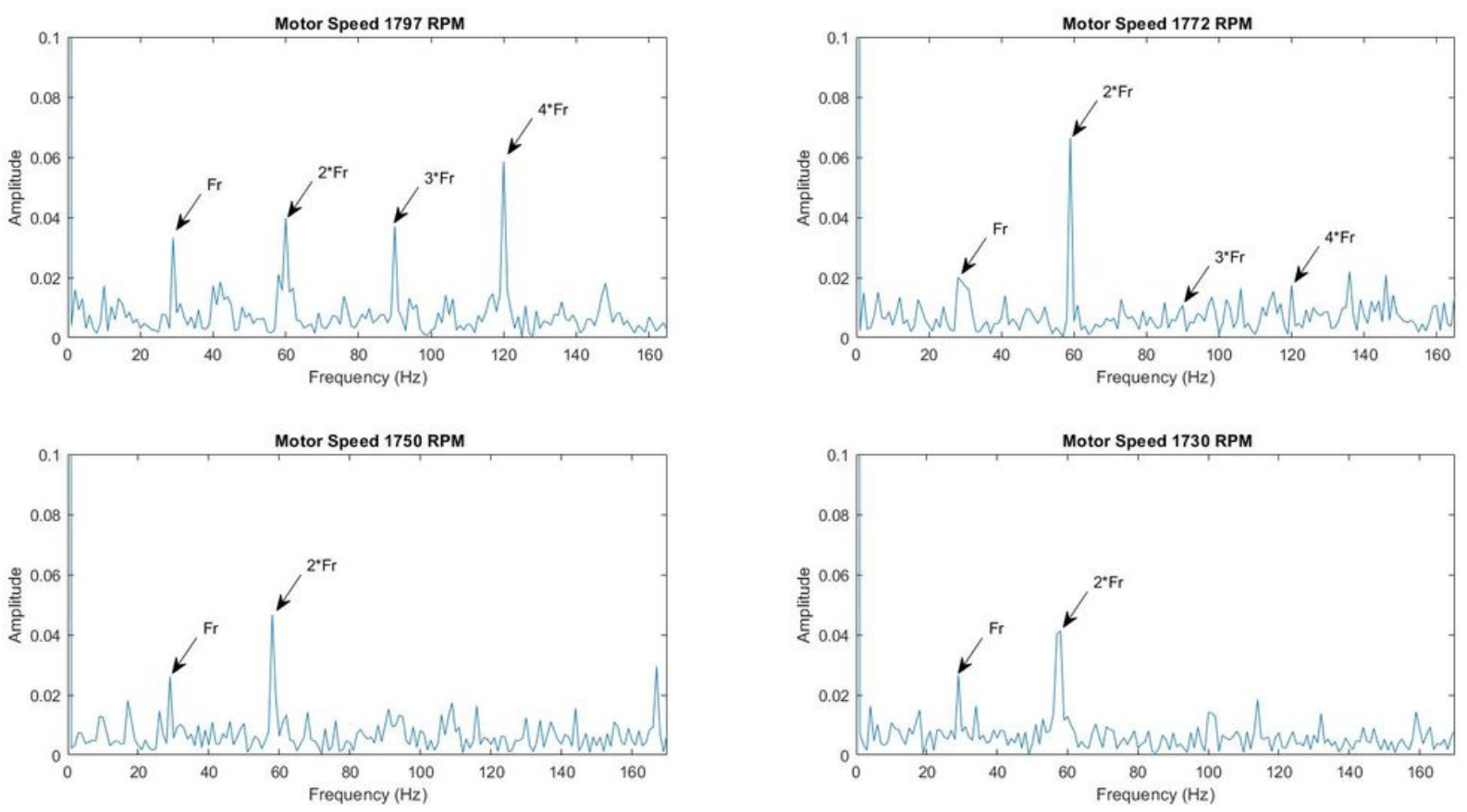

Figure 20

Envelope Spectra analysis of healthy bearing signal by the proposed method. 